\title{
Two-stage MINLP algorithm for the optimal synthesis and design of networks of CHP units
}

\author{
Cristina Elsido ${ }^{\text {a, }}$, Aldo Bischi ${ }^{b}$, Paolo Silva ${ }^{\text {a }}$, Emanuele Martelli a, ** \\ a Politecnico di Milano, Department of Energy, Via Lambruschini 4, 20156 Milano, Italy \\ ${ }^{\mathrm{b}}$ Skolkovo Institute of Science and Technology, Moscow, Russian Federation
}

\begin{abstract}
This paper proposes a Mixed Integer Non Linear Programming (MINLP) model and two-stage optimi-zation algorithm for determining the most profitable synthesis and design of Combined Heat and Power units within a district heating network with heat storage while taking into account the optimal scheduling of the units over the year. A two-stage algorithm for tackling the challenging MINLP problem is devised: at the upper level the selection and sizing of the units is optimized by means of specifically selected evolutionary algorithms, while at the lower level the operational scheduling problem is line-arized and optimized with a commercial Mixed Integer Linear Programing solver. Three different approaches, based on two different evolutionary algorithms and discrete variable relaxation, are devised and compared to tackle the upper level problem. Moreover a bounding technique is proposed to limit the computational time required to solve the lower-level problem. The overall algorithm is tested on an industrial scale problem to find the two system designs leading to the minimum energy consumption and the minimum total annual cost. Computational results indicate that the continuous relaxation of the plant sizes significantly helps to improve the convergence rate of the tested evolutionary algorithm and to find improved solutions. For the considered test case, the design optimized for the minimum energy consumption allows to save 64\% of primary energy compared to the minimum total annual cost solution, but with a $28 \%$ higher total annual cost.
\end{abstract}

\section{Introduction}

Combined Heat and Power (CHP) plants, also called cogeneration plants, are energy systems that convert primary energy (e.g., fossil fuels) into electricity and useful heat in order to satisfy the electricity and thermal demands of a set of users. Cogeneration is an effective thermodynamic expedient to achieve energy, money and emission savings [1] through the improved exploitation of the primary energy used by engines and power cycles. The increased first principle efficiency (sum of the electric and thermal efficiency) and achievable fuel saving make them an attractive option for both small (domestic) [2] and large (industrial) applications [3] with considerable fuel savings compared to the use of standard (separated) generation units (i.e., a boiler for the generation of heat and a

\footnotetext{
* Corresponding author.

** Corresponding author.

E-mail addresses: cristina.elsido@polimi.it (C. Elsido),

F-emanuele.martelli@polimi.it (E. Martelli).
}

power plant for the generation of electric power).

Furthermore, it is possible to enhance the performance of CHP plants by adding thermal storage systems [4] which allow to uncouple the operation of the CHP unit from the profile of thermal power demand. Indeed, if a thermal storage tank is included, heat generated by the units can be temporarily stored by allowing a certain shift between the heat generation time and the thermal demand. Such a possible time shift can be exploited in the following ways:

- switch on the CHP units at full load when the electricity selling prices are high so as to maximize the economic revenue [5];

- operate the CHP units mainly at full load (and then switching it off when it is not necessary to charge the storage system), which typically corresponds to the maximum efficiency operating point [4];

- adopt smaller CHP units, with size close to the average value of heat demand profile. Without heat storage system it would be necessary to size the CHP units for the peak value of the heat demand with an increase in capital costs; 
- Reduce the number of on/off switches of the units (both CHP units and boilers) which have inefficiencies associated to the start-up/shut-down phase.

All the above-listed advantages lead to a considerable decrease of primary energy consumption and $\mathrm{CO}_{2}$ emissions, as shown in Refs. [6] and [5].

In order to increase the operational flexibility (i.e., maximum and minimum load) and reliability of the heat generation system as well as to exploit the possible synergies between the different units, multiple generation units (even of different sizes and types, such as CHP engines, CHP gas turbines, CHP steam cycles, boilers, heat pumps, etc.) are typically used. These units are connected to the thermal storage system and the end users via a heat distribu-tion network typically using pressurized hot water as heat carrier. The typical supply temperature is in the range $100-120^{\circ} \mathrm{C}$ and return temperature of about $65-85^{\circ} \mathrm{C}$, as deployed in the Turin's district heating network [7]. Pressurized water is commonly adopted also in the so called second generation and third genera-tion district heating networks with supply temperature even below $100^{\circ} \mathrm{C}$ [8].

A key issue to make CHP systems (and specially networks of CHP and generation units) competitive in terms of economic revenues, primary energy savings and emissions is the careful optimization of the design and operation [9]. The design of CHP systems and networks of CHP and generation units is particularly challenging because it is necessary to select the type of units to install as well as their size taking into account two conflicting energy performance indexes (i.e., electric efficiency and thermal efficiency), the economic profitability indexes, as well as their operational flexibility (scheduling limitations as well as part-load performance and influence of the ambient temperature). Con-cerning the last point, it is very important to consider the operation and scheduling of the CHP and generation units already in the design phase taking into account the expected profiles of power and thermal demands over the whole operating horizon (at least one year so as to take into consideration sea-sonal variations), expected profiles of electricity prices, technical features of the commercially available (co)generation units (available types, available sizes, performance maps, start-up and shutdown behavior), as well as the seasonal profile of the ambient temperature (which influences the performance of gas turbines, heat pumps and engines). Thus, in order to properly consider the expected operation and scheduling of the units (hence, a good estimate of the actual flexibility limits, part-load performance and operating costs), the design optimization problem must include the multiperiod scheduling problem [10] for a set of most relevant operating periods (e.g., typical days [11]). The resulting design problem is a challenging Mixed Integer Non Linear Program (MINLP), as shown in Section 2. The solution of this problem requires ad hoc approaches as general purpose MINLP solvers cannot even solve the plain scheduling subproblem [12]. Simplified sizing methods, like that proposed in Ref. [13] are not suitable because they consider systems based on a single CHP unit and not a network featuring multiple CHP units.

A thorough review of the approaches and algorithms proposed in the last 20 years for tackling the MINLP design optimization problem including scheduling is reported in Section 3. Despite the attractiveness of the global optimality guaranteed by the problem linearity and the effectiveness of the available commercial solvers for Mixed Integer Linear Problems (e.g., CPLEX, GUROBI, etc.), linearization approaches (reviewed in Section 3.1) cannot consider an extended set of typical operating periods (due to the necessity of taking into account all the time periods at once in a single MILP) and, for units with continuous size, cannot accurately model the actual nonlinear effects of the size on the performance maps of the (co)generation units. On the other hand, decomposition approaches (see Section 3.2) rely on meta-heuristic algorithms (e.g., evolutionary algorithms) for the selection of the units and their sizes which suffer from poor convergence rates (hence long computational times) and the lack of convergence guarantees. Thus, devising an efficient algorithm for tackling the MINLP design optimization problem of complex networks of CHP units is still an open challenge.

In this paper, we adopt the problem decomposition approach and propose an efficient two-stage algorithm for tackling the design optimization problem of CHP systems and networks of CHP units with and without heat storage. In Section 2, the overall problem related to the synthesis/design and scheduling of networks of CHP units is formulated as a Mixed Integer Non Linear Programming (MINLP) problem. In Section 3, the main solution approaches available in the scientific literature are critically reviewed. In Section 4, the two-level decomposition approach and related optimization algorithms are described, while in Section 5 a systematic and optimized framework for selecting representative operating periods (i.e., weeks) is presented. In Section 6, the overall methodology is applied to a real-world test case to show its effectiveness.

\section{Problem statement and challenges}

The optimization problem related to the synthesis and design of networks of CHP units for multi-period operation can be stated as follows.

Given:

- the expected hourly (time-dependent) profiles of heat and electricity demands for the whole operating time horizon (typically one year, so as to take into consideration seasonal variations);

- the expected hourly time-dependent prices for purchasing and selling electricity from/to the electric grid;

- the expected fuel costs;

- the average seasonal profile of ambient temperature at the installation site;

- a catalog of potentially available (co)generation units with discrete sizes (e.g. gas turbines, internal combustion engines);

- a list of potentially available (co)generation units with continuous (customized) sizes (e.g. boilers, steam cycles);

- a potentially available heat storage system with continuous size;

- the expected lifetime of the network of CHP units and the relevant economic parameters (e.g., return on investment, income and property tax, book depreciation, etc.),

\section{determine:}

- which (co)generation units to install;

- whether or not installing the storage system;

- the sizes of the selected units and storage system,

so as to minimize the a relevant objective function, while satisfying the demands of heat and electricity during every expected time period.

It is necessary to determine the optimal scheduling of the network of CHP units over the whole operating time horizon $T$ in order to take into account the scheduling limitations as well as part-load performance of the selected units. In particular, the following variables must be determined for each time period: 
- the on/off statuses of selected units;

- the values of the operative variables of selected units;

- the use of the heat storage system.

The superstructure of the network of CHP units with an example of possible configurations is represented in Fig. 1. Electric energy can be produced by the power (co)generation units (i.e. gas turbines or internal combustion engines in Fig. 1) and delivered to the user or supplied to electricity users, or it can be purchased/sold from/to the grid. Thermal energy can be produced by the heat (co)generation units or by auxiliary boilers and, possibly, accumulated in storage tanks, in order to meet the heat users' demands.

Heat, power and cogeneration units must be classified on the basis of two main criteria, the first one concerning their design and the second one concerning their operation:

- their size availability on the market, either discrete (as for gas turbines and internal combustion engines), or continuous as for industrial boilers and steam cycles (specifically designed for the application);

- their number of independent operative variables, also called "degrees-of-freedom", which allow to control the heat and po-wer output [10].

The above-stated problem can be formulated as a Mixed Integer Non-Linear Program (Problem P1). The objective function of the optimization problem is the Total Annual Cost (TAC):
In Eq. (1), $z_{\mathrm{D}, i}, x_{\mathrm{D}, i}$ and $y_{\mathrm{D}, i}$ denote the variables for synthesis and design optimization, the variables $z_{\mathrm{O}, i}, x_{\mathrm{O}, i}$ are the variables for scheduling and operation optimization. In particular, the binary variables $z_{\mathrm{D}, i}$ denote the selection of the $i$-th type of CHP unit (e.g., gas turbine, engine, etc.), while the discrete variable $y_{\mathrm{D}, i}$ allow to select the unit size among the commercially available ones (units in set $C A$ ), and the real variable $x_{\mathrm{D}, i}$ is defined for the units with customizable size (units in set $C U$ ). The variables $z_{0, i}, x_{0, i}$ are the independent variables for scheduling and operation optimization: $z_{\mathrm{O}, i}$ denotes the on/off status of components, while $x_{O, i}$ denotes the continuous independent operation control variables (e.g., load). The continuous (real) variables $l_{H, \text { storage }}$ and $u_{\mathrm{H}, t}$ label respectively the maximum capacity and the level of heat accumulated in the storage system.

$\operatorname{TAC}\left(z_{\mathrm{D}, i}, x_{\mathrm{D}, i}, y_{\mathrm{D}, i}, z_{0, i, t}, x_{\mathrm{O}, i, t}, u_{\mathrm{H}, t}, l_{\mathrm{H}, \text { storage }}\right)$ is the total annual cost, the objective function of the optimization problem. It includes the operating and maintenance costs of the energy sys-tems (first two terms of the formula of $T A C$ ) over the whole plant lifetime $T$, and the annualized cost of investment (last term). In particular, $C_{O \& M, t}$ are the operational costs (e.g. fuel, operation and maintenance, start-up) of all units of the system at time $t, R_{t}$ are the revenues from selling electricity at time $t, C_{i n v, i}$ and $C_{i n v, \text { storage }}$ are the investment costs for unit $i$ and for the storage and CC is the capital carrying charge rate [14], that takes into account the interest on the invested funds as well as other incidental costs. The exponential economy-of-scale law [15] is assumed to model the effect of the size on the capital cost of the

$$
\begin{aligned}
\min _{z_{\mathrm{D}, i}, x_{\mathrm{D}, i}, y_{\mathrm{D}, i}, z_{0, i, t}, x_{\mathrm{O}, i, t}, u_{\mathrm{H}, t}, l_{\mathrm{H}, \text { storage }}} T A C= & \sum_{t \in T} C_{O \& M, t}\left(z_{\mathrm{D}, i}, x_{\mathrm{D}, i}, y_{\mathrm{D}, i}, z_{\mathrm{O}, i, t}, x_{\mathrm{O}, i, t}, u_{\mathrm{H}, t}, l_{\mathrm{H}, \text { storage }}\right)-\sum_{t \in T} R_{t}\left(z_{\mathrm{D}, i}, x_{\mathrm{D}, i}, y_{\mathrm{D}, i}, z_{O, i, t}, x_{\mathrm{O}, i, t}, u_{\mathrm{H}, t}, l_{\mathrm{H}, \mathrm{storage}}\right) \\
& +C C \cdot \sum_{i \in \text { All }} C_{i n v, i}\left(z_{\mathrm{D}, i}, x_{\mathrm{D}, i}, y_{\mathrm{D}, i}\right)+C C \cdot C_{i n v, \text { storage }}\left(l_{\mathrm{H}, \text { storage }}\right)
\end{aligned}
$$

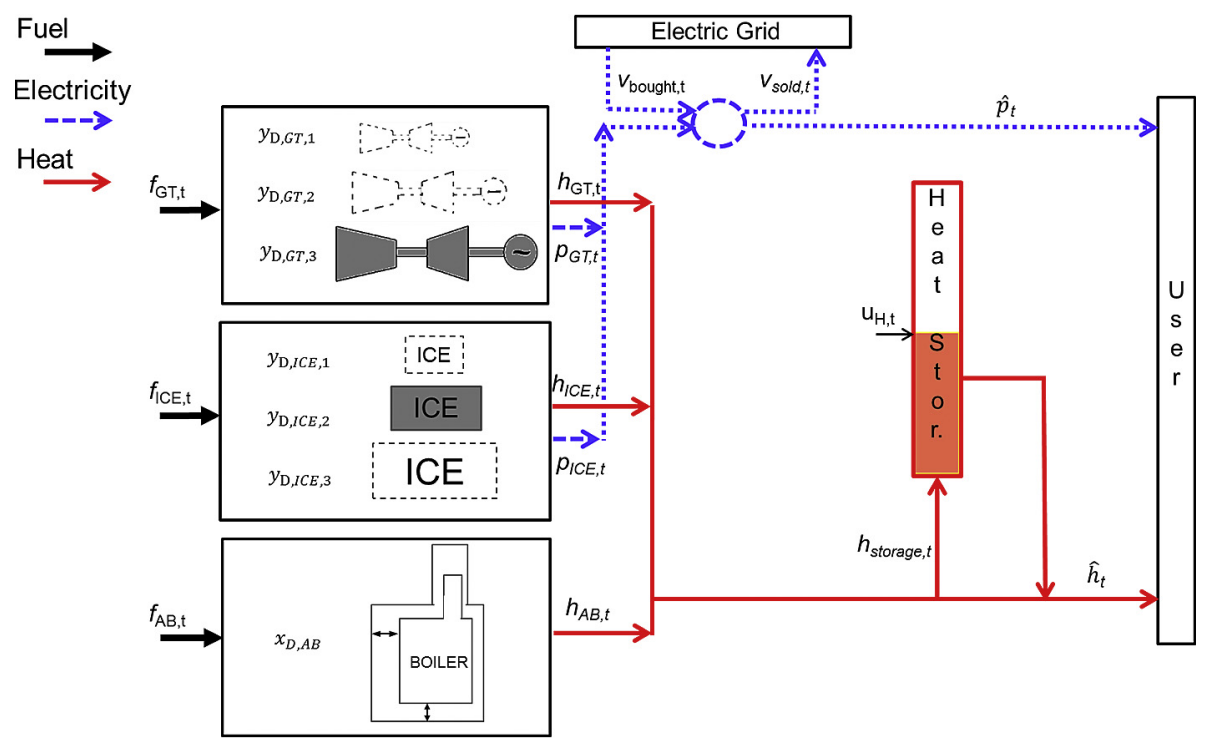

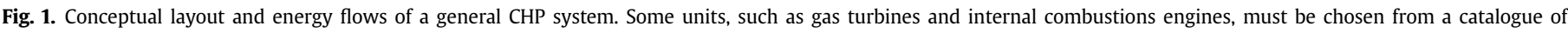

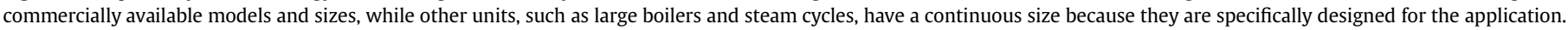


units with variable size, as indicated in Eq. (2),

$C_{i n v, i}\left(z_{\mathrm{D}, i}, x_{\mathrm{D}, i}\right)=z_{\mathrm{D}, i} \cdot C_{i n v, i}^{r e f} \cdot\left(\frac{x_{\mathrm{D}, i}}{x_{\mathrm{D}, i}^{r e f}}\right)^{s_{i}} \quad \forall i \in$ All.

In Eq. (2) $C_{i n v, i}^{r e f}$ denotes the investment cost of a unit with reference size $x_{\mathrm{D}, i}^{r e f}$. $s_{i}$ is the exponent of the scale law (typically ranging between 0.6 and 0.9 depending on the type of energy system [16]).

The constraints of the problem are described in the following Eq. (3)-(24).

\section{Constraints on the design variables}

$g_{\mathrm{D}}\left(z_{\mathrm{D}, i}, x_{\mathrm{D}, i}, y_{\mathrm{D}, i}\right) \leq 0$

Eq. (3) represents possible constraints on the design variables (i.e., not involving operation variables) that may be related to technical or economical limitations, expressed typically as inequalities (e.g., minimum or maximum allowed size of the network of CHP units, maximum total number of units to be installed, maximum allowed investment cost, etc.).

Logical constraints between design and operation variables

$z_{\mathrm{O}, i, t} \leq z_{D, i} \quad \forall i \in A l l, \forall t \in T$

Eq. (4) is a logical constraint linking the binary selection variables to the binary scheduling variables. These constraints guarantee that in each period of time only the selected units (i.e., selected to be installed on the system) can be switched on and used.

Performance curves of units with discrete size (Eqs. (5)-(7)) and units with customizable size (Eqs. (8)-(10))

$$
\begin{array}{cc}
f_{i, t}=z_{0, i, t} \phi_{\mathrm{F}, i}\left(x_{\mathrm{O}, i, t}, y_{\mathrm{D}, i}\right) & \forall i \in C A, \forall t \in T \\
p_{i, t}=z_{\mathrm{O}, i, t} \phi_{\mathrm{P}, i}\left(x_{\mathrm{O}, i . t}, y_{\mathrm{D}, i}\right) & \forall i \in C A, \forall t \in T \\
h_{i, t}=z_{\mathrm{O}, i, t} \phi_{\mathrm{H}, i}\left(x_{\mathrm{O}, i, t}, y_{\mathrm{D}, i}\right) & \forall i \in C A, \forall t \in T \\
f_{i, t}=z_{\mathrm{O}, i, t} \phi_{F, i}\left(x_{\mathrm{O}, i, t}, x_{\mathrm{D}, i}\right) & \forall i \in C U, \forall t \in T \\
p_{i, t}=z_{\mathrm{O}, i, t} \phi_{\mathrm{P}, i}\left(x_{\mathrm{O}, i, t}, x_{\mathrm{D}, i}\right) & \forall i \in C U, \forall t \in T \\
h_{i, t}=z_{\mathrm{O}, i, t} \phi_{\mathrm{H}, i}\left(x_{\mathrm{O}, i, t}, x_{\mathrm{D}, i}\right) & \forall i \in C U, \forall t \in T
\end{array}
$$

The constraints in Eqs. (5)-(7) and Eqs. (8)-(10) represent the performance curves of the units. The variables $f_{i, t}, p_{i, t}, h_{i, t}$ are the fuel input and energy output (electric power and heat) of (co) generation units. The functions $\phi_{F, i, t}, \phi_{P, i, t}, \phi_{H, i, t}$ denote the performance curves of the units relating the unit size and the independent operation variables $x_{0, i, t}$ to the fuel input $f_{i, t}$, electric power output $p_{i, t}$ and thermal power output $h_{i, t}$. Such functions must be derived from experimental data or provided by the unit's manufacturer. They are typically nonlinear with respect to both unit size and operation control variables (as shown in Ref. [10]). The effect of the size on the efficiency of power plants and engines is due to two factors: the positive effects of having larger sizes on the isentropic efficiency of turbomachines (tur-bine, pumps, compressors) due to the lower fluid friction losses [17] and the positive effects on specific costs (economy-of-scale law) which allow to use larger heat transfer areas, more expensive materials and/or technologies for large power plants. Steam cycles are affected by a significant size effect on the isentropic efficiency of the turbine stages which varies from $60 \%$ to $92 \%$ depending on the stage size parameter, as shown by Lozza [18]. According to the statistical analysis shown in Ref. [16], also the efficiency of gas turbines are strongly influenced by the size. Medium-small gas turbines ( $<100 \mathrm{MW}$ electric power) have on average a size effect on the heat rate (i.e., fuel thermal input divided the net electric power generate) following the scale law with an exponent of 0.89 , from an average value of electric efficiency $28.5 \%$ at $5 \mathrm{MW}$ to more than $41 \%$ at $100 \mathrm{MW}$. Large gas turbines ( $>100 \mathrm{MW}$ ) and natural gas combined cycles show a lower but still non-negligible size effect on the performance [16]. According to the data reported on catalogues of boilers (see e.g., [19]), also the thermal efficiency of gas-fired industrial boilers for hot water production increases with size, going from $90 \%$ at $1 \mathrm{MW}$ to almost $93 \%$ at $20 \mathrm{MW}$.

As for the effect of the operation control variables, for some units, like boilers and heat pumps, the performance curves are typically concave functions with respect to the control variables $x_{0, i, t}$ (thus an advantage for the linearization of the optimization problem, as shown in Ref. [10]), while for units like CHP gas turbines the performance curves are typically non-concave and even non-smooth (due to the switch of part-load control strategy [10]). Besides, the performance of gas turbines, engines and heat pumps are strongly affected by the ambient temperature, thus the performance curves of these units depend on the ambient temperature at time $t$ (as indicated by the subscript $t$ in the functions $\left.\phi_{F, i, t}, \phi_{P, i, t}, \phi_{H, i, t}\right)$. An example of the influence of the ambient temperature on the performance map of a gas turbine is reported in Section 4.1.

In Eqs. (5)-(10), the performance maps are multiplied by $z_{0, i, t}$ so as to guarantee that if the unit is switched off $\left(z_{0, i, t}=0\right)$, the fuel input and power output are zero.

Heat and electricity balances

$\sum_{i \in \text { All }} h_{i, t}+\left(1-u_{\mathrm{H}, \mathrm{losses}}\right) \cdot u_{\mathrm{H}, t}-u_{\mathrm{H}, t+1}=\widehat{h}_{t} \quad \forall t \in T$

$v_{\text {sold }, t}-v_{\text {bought }, t}=\sum_{i \in \text { All }} p_{i, t}-\widehat{p}_{t} \quad \forall t \in T$

Eq. (11) is the heat balance of the overall network including thermal storage system. It guarantees that in each period of time $t$ the heat produced by all units and the heat in the storage system at time $t$, except for the heat losses, are equal to the heat demand of the users plus the heat in the storage system at the beginning of the following period of time.

Eq. (12) is the electricity balance of the overall network. The variables $v_{\text {sold, } t}$ and $v_{\text {bought }, t}$ indicate respectively the electricity sold to or bought from the electric grid. The constraint guarantees that in each period of time $t$ the sum of the electricity produced by all power and cogeneration units and the electricity bought from the electric grid is equal to the sum of the heat demand of the users and the electricity sold to the electric grid.

\section{Constraints on the storage capacity}

$$
\begin{aligned}
& u_{\mathrm{H}, t} \leq l_{\mathrm{H}, \text { storage }} \quad \forall t \in T \\
& u_{\mathrm{H}, T_{\max }}=u_{\mathrm{H}, 1}
\end{aligned}
$$

Eq. (13) is an upper limit on the amount of heat stored in the system, guaranteeing that the heat stored in the thermal storage 
never exceeds the maximum storage capacity. Eq. (14) is an equality constraint needed to keep the problem consistent if typical operating periods (e.g., typical days or weeks) are considered to simulate the expected lifetime of the system $T$ : it avoids that, in each typical day/week, the system uses the heat initially available in the storage system without refilling it to the same level.

\section{Bounds on the variables}

$$
\begin{aligned}
& X_{\mathrm{D} \min , i} \leq x_{\mathrm{D}, i} \leq X_{\mathrm{D} \text { max }, i} \quad \forall i \in C U \\
& X_{\mathrm{O} \min , i} z_{\mathrm{O}, i} \leq x_{\mathrm{O}, i, t} \leq X_{\mathrm{O} \text { max }, i} z_{\mathrm{O}, i} \quad \forall i \in A l l, \forall t \in T \\
& l_{\mathrm{H}, \text { storage,min }} \leq l_{\mathrm{H}, \text { storage }} \leq l_{\mathrm{H}, \text { storage } \text { max }}
\end{aligned}
$$

Eq. (15) defines the upper and lower bounds on the size of each unit with customizable size. Eq. (16) guarantees that the continuous operation control variables $\left(x_{O, i}\right)$ are set to 0 when the component is switched off (i.e., $z_{\mathrm{O}, i}=0$ ), and that they are bounded between the minimum and maximum load when the component is switched on (i.e., $z_{\mathrm{O}, i}=1$ ). Eq. (17) defines the upper and lower bounds on the capacity of the heat storage system.

Definition of the variables

$$
\begin{aligned}
& y_{\mathrm{D}, i} \in\left\{\mathrm{Y}_{i, 1}, Y_{i, 2}, Y_{i, 3}, \ldots\right\} \quad \forall i \in C A \\
& x_{\mathrm{D}, i} \geq 0, \quad x_{\mathrm{D}, i} \in \mathbb{R} \quad \forall i \in C U \\
& z_{\mathrm{D}, i} \in\{0,1\} \quad \forall i \in A l l \\
& z_{\mathrm{O}, i, t} \in\{0,1\} \quad \forall i \in A l l, \forall t \in T \\
& l_{\mathrm{H}, \text { storage }} \geq 0, \quad l_{\mathrm{H}, \text { storage }} \in \mathbb{R} \\
& f_{i, t}, p_{i, t}, h_{i, t} \geq 0 \quad f_{i, t}, p_{i, t}, h_{i, t} \in \mathbb{R} \quad \forall i \in A l l, \forall t \in T \\
& u_{\mathrm{H}, t}, v_{\text {sold }, t}, v_{\text {bought }, t} \geq 0 \quad u_{\mathrm{H}, t}, v_{\text {sold }, t}, v_{\text {bought }, t} \in \mathbb{R} \quad \forall t \in T
\end{aligned}
$$

Eq. (18-24) defines the type (either real, real positive or binary) of all the variables of the problem. Eq. (18) states that the $y_{\mathrm{D}, i}$ can assume only the discrete values $Y_{i, 1}, Y_{i, 2}, Y_{i, 3}$, etc. Eqs. (19) and (22-24) indicate that $x_{\mathrm{D}, i}, l_{\mathrm{H}, \text { storage }}, f_{i, t}, p_{i, t}, h_{i, t}, u_{\mathrm{H}, t}, v_{\text {sold }, t}, v_{\text {bought }, t}$ are real positive variables, while Eqs. (20) and (21) indicate that $z_{\mathrm{D}, i}$ and $z_{0, i, t}$ are binary.

Problem (P1) is defined by Eqs. (1-24).

The formulation can be extended to model energy networks of units with multiple types of energy demands (e.g., heat at different temperature levels or cooling load, as done in Bischi et al. [10]), or to add start-up and ramp-up constraints (e.g., [20]).

Due to the presence of the non-linear constraints corresponding to the performance curves of generation units (typically nonlinear with respect to load as well as size), the design of CHP energy systems is a Mixed Integer Non-Linear Programming (MINLP) problem.

\section{Relevant literature}

The MINLP problem (P1) is extremely challenging due to its nonlinearity, potential non-convexity and large number of variables brought about by the multi-period operational scheduling problem. Tackling (P1), even with state-of-the-art algorithms, would be extremely challenging even for small scale instances with a few units: as shown by Taccari et al. (2015) [12], state-ofthe-art MINLP algorithms are not able to solve just the optimal scheduling sub-problem (where design variables are fixed). For this reason, different authors have proposed either linearization approaches or decomposition strategies and ad hoc solution algorithms.

\subsection{Linearization approaches and MILP formulations}

The operations research state of the art approach consists in linearizing the original MINLP problem so as to obtain a Mixed Integer Linear Problem (MILP) which can be tackled more easily. Compared to MINLP problems, linearizing the problem and tackling a MILP lead to the following advantages:

- there are convergence guarantees on the solution (the returned solution is globally optimal, without the risk of finding a local optimum, and the solution accuracy is indicated by the branch and bound gap [21]),

- the MILP can be tackled by extremely fast and effective commercially available MILP solvers (e.g., CPLEX [22], Gurobi [23], Xpress [24]).

For instance, Iyer and Grossmann (1998) [25] proposed a MILP model for the synthesis and operational planning of utility systems for multi-period operation with varying demands. The objective was to minimize the sum of total operating costs, summed over all periods of operation, and the amortized investment cost of equipment. The formulation included binary variables both for the selection and for operational status of units during each period, and continuous variables for the sizes and the operation variables. The authors proposed a decomposition method to reduce the computational complexity of the problem: in the higher level the system configuration is chosen, by relaxing the operational integer variables and solving a MILP problem with only a few binary variables. Then, for fixed design binary variables, the operational problem and the sizes of selected components are optimized, by solving a MILP sub-problem. Different system configurations are generated and evaluated by adding an integer and design cut in the design problem at the end of each iteration. The yearly variations in energy demand are considered using 12 periods, corresponding to the months. It is worth noting that the optimal scheduling problem cannot be solved separately for each time period, due to the linking constraints that relate operational variables to design variables.

Maréchal and Kalitventzeff (2003) [26] proposed a MILP model for the synthesis of multi-period utility systems which must be thermally integrated in the chemical process. They combined the MILP model proposed by Iyer and Grossmann (1998) [25] for the optimization of the synthesis and operational planning of utility systems with the multi-period heat cascade constraints presented in Maréchal and Kalitventzeff (1998) [27]. To keep the formulation linear, performance curves and costs of units are assumed to be linear or piecewise linear with respect to the real variables (sizes of units and loads). Authors do not specify how the operating periods have been defined.

Arcuri et al. (2007) [28] developed an iterative procedure for the optimal design and sizing of a trigeneration plant, proposed to meet the energy demands of the customer. The types of units and their sizes are fixed by an external iterative procedure, while the operating costs of the sampled designs are evaluated by solving a MILP problem related to the optimal scheduling over six 
typical days. The length of time intervals is $2 \mathrm{~h}$ so as to limit the problem size.

Yokoyama et al. (2002) [29] proposed a MILP model and decomposition strategy to simultaneously optimize the synthesis and scheduling of energy supply systems with continuous sizes. In order to preserve the linearity of the problem, the authors assumed that the performance curves are linear (or piecewise linear) with respect to unit loads and that they can only be shifted by a quantity that depends linearly on the unit's size. Moreover, cost functions (investment and operating costs) are linearized. Also the maximum and minimum loads are assumed to vary linearly with the sizes of the units. To consider seasonal and hourly variations in energy demands, the typical year is divided into 3 representative days, one for the summer, one for the winter and one for the mid-season, with time periods ranging from 1 to $4 \mathrm{~h}$. Due to the necessity of simultaneously tackling the synthesis and scheduling problem for the whole time horizon, the resulting MILP turns out to be quite challenging because of the large number of variables and constraints. For this reason the authors proposed a decomposition method that takes advantage of the block angular structure of the constraint matrix to find a close-to-optimal solution in a short computational time.

Yokoyama et al. (2006) [30] revised the MILP formulation originally proposed [29] to deal with units (e.g. gas turbines) with discrete sizes. The authors introduce a binary variable to denote the selection of the type and size of the units and an integer variable $(0$, $1,2, \ldots, N$ ) to define the number of units (of the same type and size). Each type of unit may have a different performance curve depending on the size, as far as it is linear (or piecewise linear). It is assumed that the units of the same type and size must be operated at the same load level. As commercial MILP solvers are not capable of finding the optimal solution for industrial scale problem within reasonable time, Yokoyama et al. (2015) [31] have recently proposed a branch and bound method which takes advantage of the hierarchical relationship of design and operation variables.

Among the above-listed MILP linearization approaches, the models proposed by Yokoyama et al ([29] and [30]). appear to be the most general ones. However, as far as the model proposed in Yokoyama et al. [30] is concerned, it is important to point out an important limitation: the assumption that the units of the same type and size must be operated at the same load level. Since units like gas turbines and internal combustion engines have considerable performance decay at part loads, it is more advantageous to operate one unit at full load while keeping the other units switched off. For this reason, the limitation of operating the units of the same type and size at the same part loads may lead to sub-optimal solutions. It is worth noting that, within the model proposed by Yokoyama et al. ([29] and [30]), this assumption cannot be avoided since only one operational variable is defined for the units of the same type and size, so as to minimize the number of variables. To obtain such improved operational flexibility, it is necessary to introduce one binary selection variable and one binary on/off variable for each unit of the same type and size, with an increase of the problem size.

Aguilar et al. (2007) [32] presented a method that combines elements of both operational and design problems in a unique MILP problem. The authors optimize units selection as binary variables, equipment sizes as continuous variables and operational decisions as binary and continuous variables. The design and operational variables are optimized simultaneously under different operating scenarios: the design variables connect different time period or scenarios, which are treated as single time intervals with separate operational variables. This approach is not suitable for modeling time-linking constraints such as ramp rates or heat storage systems. The authors presented two test cases with 24 periods ( 12 seasonal periods further sub-divided into two inter-seasonal ones).

A similar MILP approach for optimizing simultaneously the synthesis, design and operation optimization of energy supply systems was proposed by Buoro et al. (2013) [33]. In this formulation, binary variables represent selection and on/off status of units, while all other variables are continuous. Linear approximations of performance curves of units were considered. The authors used twelve typical weeks, each of $168 \mathrm{~h}$, to represent the year.

Omu et al. [34] devised a MILP model for the design of CHP systems and related energy distribution networks to meet the electricity and heat demands of a cluster of commercial and residential buildings. The proposed MILP model allows to optimize the selection of the technology and number of units (of fixed ca-pacity) to purchase for each site, the number of units in operation and their total load in each time period/season and site, and the necessary heat and power distribution network. The MILP model is targeted at a high-level analysis of the best technologies rather than at a detailed design of the energy system since the units' part-load performance maps are not considered and the related fuel consumption is estimated assuming a constant efficiency value.

Bracco et al. [35] proposed a similar MILP model for the optimal design of distributed CHP systems for urban areas. The heat distribution network between buildings is optimized together with the selection of the CHP units and their scheduling for a set of a few (four) typical days. In order to avoid nonlinearities, the performance maps of the units is assumed to be linear between minimum and maximum load and only units with discrete sizes are considered. The problem is formulated as a multiobjective MILP so as to identify the Pareto optimal solutions with minimum cost and minimum $\mathrm{CO}_{2}$ emissions. In Ref. [36] the authors include renewable energy sources and storage systems in the MILP model. Due to the size of the MILP and the required computational time, only three typical days are considered to assess the operation of the system.

Yang et al. (2015) [37] improved the model of Omu et al. [34] integrating it with the approach proposed by Yokoyama et al. [30] to select among units of discrete size while taking into account their part-load performance maps. Even though only 3 typical operating days (representative of the hot, cold and mid seasons) are used, the required computational time is longer than $5 \mathrm{~h}$.

In summary, the main drawbacks of MILP formulations are: (1) the large number of variables and constraints due to the necessity of taking into account all the time periods at once, (2) for units with continuous size, the impossibility of modeling the actual nonlinear effects of the unit size on the performance curve. It is important to point out that, due to the first challenge, in the above-listed MILP approaches only a few typical days (corresponding to a few tens of periods) are considered.

\subsection{Decomposition strategies}

Rather than formulating the overall design and operation problem as a single MILP problem, other authors have developed problem decomposition strategies and multi-step algorithms.

Rubio-Maya et al. (2011) [38] proposed a two-steps optimization procedure. The first step is a MINLP problem for the synthesis and design of a preliminary system on the basis of the following simplifications: (1) only one type of unit per each energy demand can be selected, (2) units have continuous sizes, (3) units may have 3 predefined operating modes (i.e., full load, heat tracking, or elec-tricity tracking modes), (4) only monthly average data of energy demands are considered. The MINLP is tackled by means of the 
DICOPT algorithm [39]. The second step, formulated as a multiperiod NLP problem and solved with the CONOPT algorithm [40], optimizes the continuous variables of design and operation using hourly data.

Fazlollahi et al. (2014) developed an optimization framework for the selection of typical operating periods [11], optimization of district energy systems with daily thermal storage [41], and the optimization of the energy distribution networks and geographical location [42]. First in Ref. [11], the energy demand profiles of the most representative operating days are determined by applying the k-means clustering algorithm. Then, in Ref. [41], at the upper level a multi-objective evolutionary algorithm optimizes the system design variables while at the lower level a multi-period energy integration MILP problem allows to optimize the scheduling of the selected units. Finally, in Ref. [42], the approach is extended to handle data, variables and constraints related to the design and operation of the distribution networks (e.g., resource locations, available pipe diameters, design flow velocity, supply and return temperatures of the heating network, etc.).

Stojiljković et al. (2014) [43] proposed a method for the synthesis, design and operation optimization of energy supply networks based on the decomposition of the problem into two subproblems: the synthesis problem and the scheduling-operation problem. The synthesis problem is combinatorial as it involves only integer variables: binary variables for the selection of a type of unit, discrete variables for selecting the type of machine model, and electric load" or "following thermal load"). Thus, the authors fix five possible "cases" (each one featuring a certain set of power generation technologies) and then optimize the sizes of the units and of the heat storage system with a multiobjective genetic algorithm. Each optimization is repeated imposing the two fixed operating modes of the units. Of course, interesting configurations (selections of the units) and operating modes risk to be overlooked as a systematic optimization of the units' selection and scheduling is not performed.

\section{Problem decomposition and two-stage optimization algorithm}

In this work, a two-stage decomposition of the MINLP Problem (P1) is proposed: the selection (synthesis) and sizing of the units is optimized at the upper level while the operational scheduling for the expected most relevant time periods is optimized at the lower level. In particular, for each fixed set of design variables $\left(z_{\mathrm{D}, i}, x_{\mathrm{D}, i}, y_{\mathrm{D}, i}, l_{\mathrm{H}, \text { storage }}\right)$, the lower level problem determines the optimal scheduling and storage management strategy $\left(z_{0, i, t}, x_{0, i, t}, u_{\mathrm{H}, t}\right)$ for the set of most representative operating periods (see Section 5 ).

The decomposed problem can be viewed as the following bilevel Problem (P2).

$$
\begin{aligned}
\min _{z_{\mathrm{D}, i}, x_{\mathrm{D}, i}, y_{\mathrm{D}, i}, z_{0, i, t}, x_{\mathrm{O}, i, t}, u_{\mathrm{H}, t}, l_{\mathrm{H}, \text { storage }}} T A C= & \sum_{t \in T} C_{O \& M, t}\left(z_{\mathrm{D}, i}, x_{\mathrm{D}, i}, y_{\mathrm{D}, i}, z_{O, i, t}, x_{\mathrm{O}, i, t}, u_{\mathrm{H}, t}, l_{\mathrm{H}, \text { storage }}\right)-\sum_{t \in T} R_{t}\left(z_{\mathrm{D}, i}, x_{\mathrm{D}, i}, y_{\mathrm{D}, i}, z_{O, i, t}, x_{\mathrm{O}, i, t}, u_{\mathrm{H}, t}, l_{\mathrm{H}, \text { storage }}\right) \\
& +C C \cdot \sum_{i \in \text { All }} C_{i n v, i}\left(z_{\mathrm{D}, i}, x_{\mathrm{D}, i}, y_{\mathrm{D}, i}\right)+C C \cdot C_{i n v, \text { storage }}\left(l_{\mathrm{H}, \text { storage }}\right) \text { s.t. }
\end{aligned}
$$

discrete variables for selecting number of units of each model. The synthesis and design problem is solved with six different metaheuristic methods (genetic algorithms, particle swarm optimization, ant colony optimization, simulated annealing, harmony search and tabu search) and results are compared to those obtained with a MILP-based synthesis-operation approach (see previous subsection). The operational problem, which is solved for each sampled system design, is formulated as a MILP problem. To represent the whole operating period, the authors considered four typical days of $24 \mathrm{~h}$ and included storage tank facilities (time-coupling constraints) in the multi-period operational problem. The decomposition approach turns out to be faster but in some runs a solution worse than that found by the MILP approach is found. Also a hybrid approach using the results of the decomposition approach to exclude the less promising design options from the MILP-based approach is proposed.

$$
\begin{aligned}
& g_{\mathrm{D}}\left(z_{\mathrm{D}, i}, x_{\mathrm{D}, i}, y_{\mathrm{D}, i}\right) \leq 0 \\
& X_{\mathrm{D} \text { min }, i} \leq x_{\mathrm{D}, i} \leq X_{\mathrm{D} \text { max }, i} \quad x_{\mathrm{D}, i} \in \mathbb{R} \quad \forall i \in C U \\
& y_{\mathrm{D}, i} \in\left\{\mathrm{Y}_{i, 1}, Y_{i, 2}, Y_{i, 3}, \ldots\right\} \quad \forall i \in C A \\
& l_{\mathrm{H}, \text { storage,min }} \leq l_{\mathrm{H}, \text { storage }} \leq l_{\mathrm{H}, \text { storage,max }} \quad l_{\mathrm{H}, \text { storage }} \in \mathbb{R} \\
& z_{\mathrm{D}, i} \in\{0,1\} \quad \forall i \in A l l \\
& z_{O, i, t}, x_{\mathrm{O}, i, t}, u_{\mathrm{H}, t} \text { solve }
\end{aligned}
$$

$\min _{z_{0, i, t}, x_{0, i, t}, u_{\mathrm{H}, t}} T O C=\sum_{t \in T} C_{O \& M, t}\left(z_{\mathrm{D}, i}, x_{\mathrm{D}, i}, y_{\mathrm{D}, i}, z_{O, i, t}, x_{\mathrm{O}, i, t}, u_{\mathrm{H}, t}, l_{\mathrm{H}, \text { storage }}\right)+-\sum_{t \in T} R_{t}\left(z_{\mathrm{D}, i}, x_{\mathrm{D}, i}, y_{\mathrm{D}, i}, z_{O, i, t}, x_{\mathrm{O}, i, t}, u_{\mathrm{H}, t}, l_{\mathrm{H}, \text { storage }}\right) s . t$.

Zidan et al. [44] avoid the challenging optimization of integer variables associated to the selection of the units and their on/off operation by considering a limited set of system configurations (called "cases") and only two possible operating modes ("following

$$
z_{\mathrm{O}, i, t} \leq z_{D, i} \quad \forall i \in A l l, \forall t \in T
$$


$f_{i, t}=z_{O, i, t} \phi_{\mathrm{F}, i, t}\left(x_{0, i, t}, y_{\mathrm{D}, i}\right) \quad \forall i \in C A, \forall t \in T$

$p_{i, t}=z_{\mathrm{O}, i, t} \phi_{\mathrm{P}, i, t}\left(x_{\mathrm{O}, i . t}, y_{\mathrm{D}, i}\right) \quad \forall i \in C A, \forall t \in T$

$h_{i, t}=z_{\mathrm{O}, i, t} \phi_{\mathrm{H}, i, t}\left(x_{\mathrm{O}, i, t}, y_{\mathrm{D}, i}\right) \quad \forall i \in C A, \forall t \in T$

$f_{i, t}=z_{\mathrm{O}, i, t} \phi_{F, i, t}\left(x_{\mathrm{O}, i, t}, x_{\mathrm{D}, i}\right) \quad \forall i \in C U, \forall t \in T$

$p_{i, t}=z_{\mathrm{O}, i, t} \phi_{\mathrm{P}, i, t}\left(x_{\mathrm{O}, i, t}, x_{\mathrm{D}, i}\right) \quad \forall i \in C U, \forall t \in T$

$h_{i, t}=z_{\mathrm{O}, i, t} \phi_{\mathrm{H}, i, t}\left(x_{\mathrm{O}, i, t}, x_{\mathrm{D}, i}\right) \quad \forall i \in C U, \forall t \in T$

$\sum_{i \in \text { All }} h_{i, t}+\left(1-u_{\mathrm{H}, \text { losses }}\right) \cdot u_{\mathrm{H}, t}-u_{\mathrm{H}, t+1}=\widehat{h}_{t} \quad \forall t \in T$

$v_{\text {sold }, t}-v_{\text {bought }, t}=\sum_{i \in \text { All }} p_{i, t}-\widehat{p}_{t} \quad \forall t \in T$

$u_{\mathrm{H}, t} \leq l_{\mathrm{H}, \text { storage }} \quad \forall t \in T$

$u_{\mathrm{H}, t+1}=u_{\mathrm{H}, 1}$

$X_{\mathrm{O} \min , i} z_{\mathrm{O}, i} \leq x_{\mathrm{O}, i, t} \leq X_{\mathrm{O} \max , i} z_{\mathrm{O}, i} \quad \forall i \in A l l, \forall t \in T$

$z_{\mathrm{O}, i, t} \in\{0,1\} \quad \forall i \in A l l, \forall t \in T$

$f_{i, t}, p_{i, t}, h_{i, t} \geq 0 \quad f_{i, t}, p_{i, t}, h_{i, t} \in \mathbb{R} \quad \forall i \in A l l, \forall t \in T$

$u_{\mathrm{H}, t}, v_{\text {sold }, t}, v_{\text {bought }, t} \geq 0 \quad u_{\mathrm{H}, t}, v_{\text {sold }, t}, v_{\text {bought }, t} \in \mathbb{R} \quad \forall t \in T$

The equations of Problem (P2) can be interpreted in analogy with the equations Eq. (1)-(24) of Problem (P1). Eq. (25) and Eq. (32) are the objective functions of the upper and lower level problems, respectively the Total Annual Cost (TAC) and the Total Operating Cost (TOC). Eq. (26) represents the constraints on the design variables (same as Eq. (3) in Problem (P1)), while Eqs. (27)-(30) define the type (real, discrete and binary) and the bounds of the design variables. Eq. (31) indicates that the values of the operation variables are computed by solving the lower level optimization problem defined by Eqs.(32)-(47) for fixed values of the design variables. Eqs. (32)-(44) are the constraints of the lower level operation problem and correspond to Eqs. (4)-(16) of Problem (P1). Eqs. (45)-(47) define the type (real, binary) and the bounds of the operation variables.

A schematic representation of the decomposition is reported in Fig. 2. It is important to notice that, at the lower level problem, minimizing the Total Operating Cost (TOC) is equivalent to minimizing the Total Annual Cost (TAC) because, within the lower level, the design variables and consequently the capital costs are fixed (hence, the difference between TAC and TOC is just a constant, equal to the capital costs). Then, since the two optimization levels (upper and lower) consider equivalent objective functions, Problem (P2) is in fact equivalent to the original MINLP problem (P1) in which design and operation variables are optimized at once. A

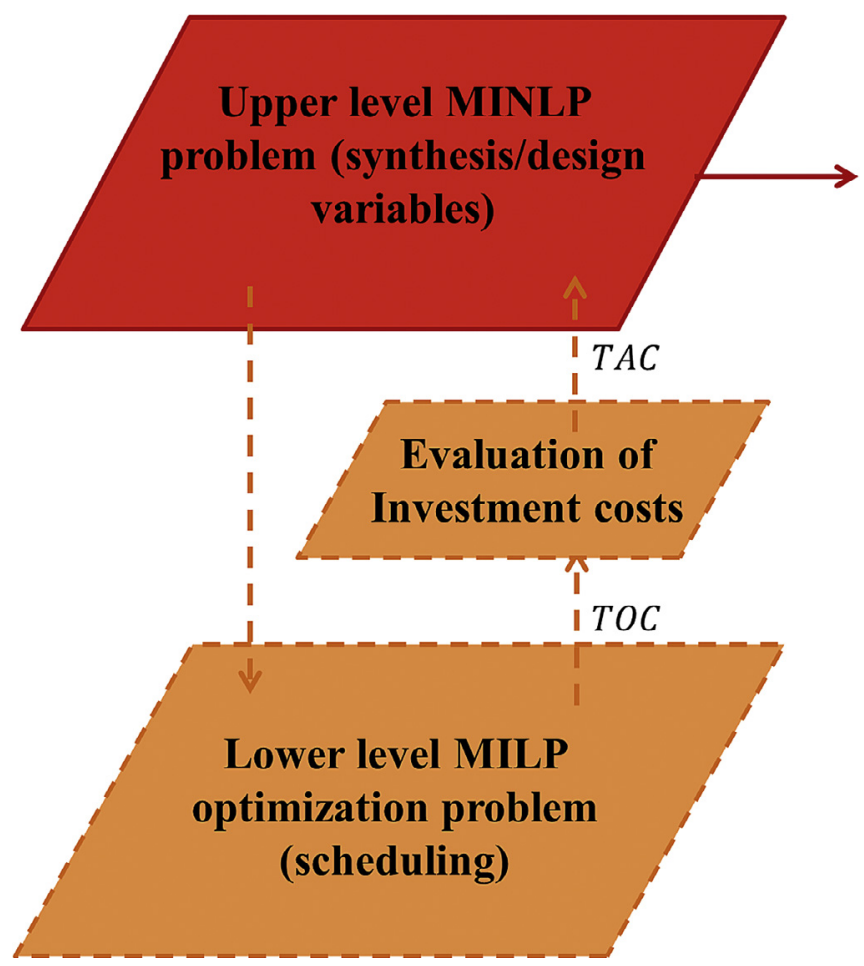

Fig. 2. Scheme of the adopted two-level decomposition. Synthesis/design variables are optimized at the upper level while the scheduling and operational variables are optimized at the lower level.

straightforward proof of the equivalence of Problem (P1) and Problem (P2) when the objective functions of the two levels are equivalent can be found in Ref. [45]. If the designer does not want to minimize the TAC but a different objective function (e.g., the primary energy consumption of the network of CHP units), he has to consider this new objective function both at the upper level and lower level problems.

\subsection{Lower level problem}

The lower level problem turns out to be the scheduling problem described and successfully tackled by several authors, such as Bischi et al. (2014) [10], Mitra et al. (2013) [20],Dvo ' r'ak et al. (2012) [46].

Given:

- the set of installed one or two-degree of freedom generation units with fixed size, and part-load performance curves;

- the heat storage tank with fixed capacity and constant loss rate;

- the expected hourly profiles of heat and electricity demands over a time horizon $T$;

- the expected hourly time-dependent prices for purchasing and selling electricity from/to the electric grid;

- the expected fuel costs;

- the average time-dependent profile of ambient temperature of the installation site,

determine for each time interval $t$ of the expected operating periods:

- the on/off statuses of units;

- the values of the operative variables of each unit;

- the storage tank levels, 
(a)

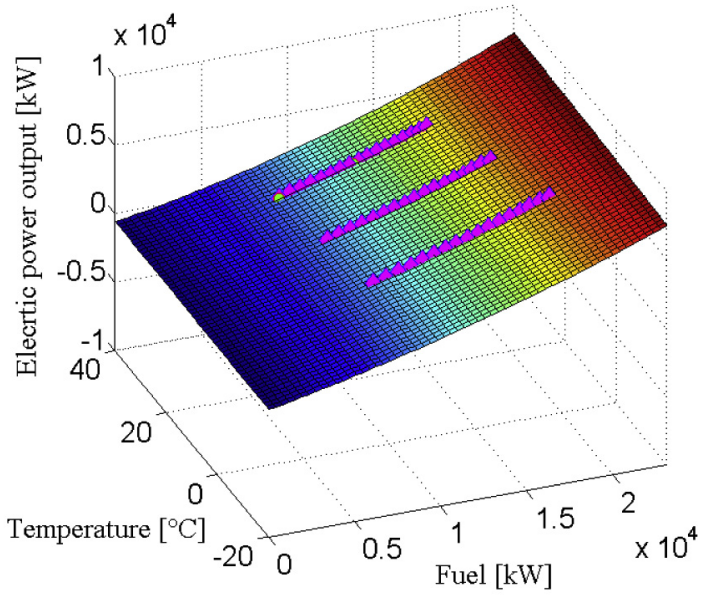

(b)

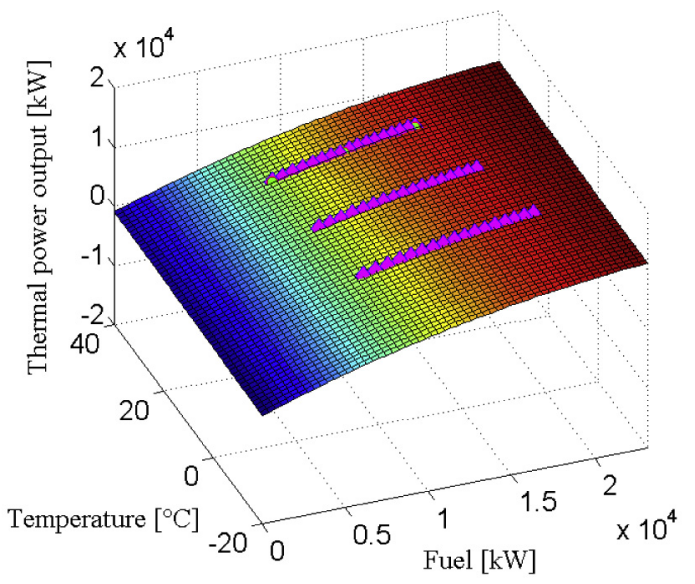

Fig. 3. Electric (a) and thermal (b) power outputs as functions of fuel and temperature for a simple cycle gas turbine with heat recovery. The purple dots on the plot label the performance maps at fixed ambient temperature (respectively $5{ }^{\circ} \mathrm{C}, 15^{\circ} \mathrm{C}$ and $25^{\circ} \mathrm{C}$ ). (For interpretation of the references to colour in this figure legend, the reader is referred to the web version of this article.)

so as to minimize the total operating cost (if the total annual cost is minimized at the upper level) or other relevant objective function, while satisfying the demands of low and high temperature heat during all the time periods.

The total operating cost includes the following terms:

- the sum of costs of the fuel utilized each hour by each unit to produce power and heat, $\mathrm{C}_{\text {fuel, }}$;

- the Operation and Maintenance (O\&M) costs which, depending on the machine, can be proportional either to the number of operating hours or to the energy produced, $\mathrm{C}_{\mathrm{O} \& \mathrm{M}, \mathrm{t}}$;

- the start-up costs which represent a penalty cost due to startup operations, $C_{\text {startup,t }}$, that can be related to fuel or power consumption and additional operation and maintenance costs;

- the sum of the revenues of the electricity sold to the electric grid whether exceeding the customer requirements $\left(\mathrm{v}_{\text {sold, } t}>\right.$ $\mathrm{v}_{\text {bought, } \mathrm{t}}$ ) and costs of the electricity bought from the grid whether the one produced is not enough to fulfill the internal requirements ( $\left.\mathrm{v}_{\text {sold, } \mathrm{t}}<\mathrm{v}_{\text {bought }, \mathrm{t}}\right), \sum_{\mathrm{t} \in \mathrm{T}} \mathrm{R}_{\mathrm{t}}$.

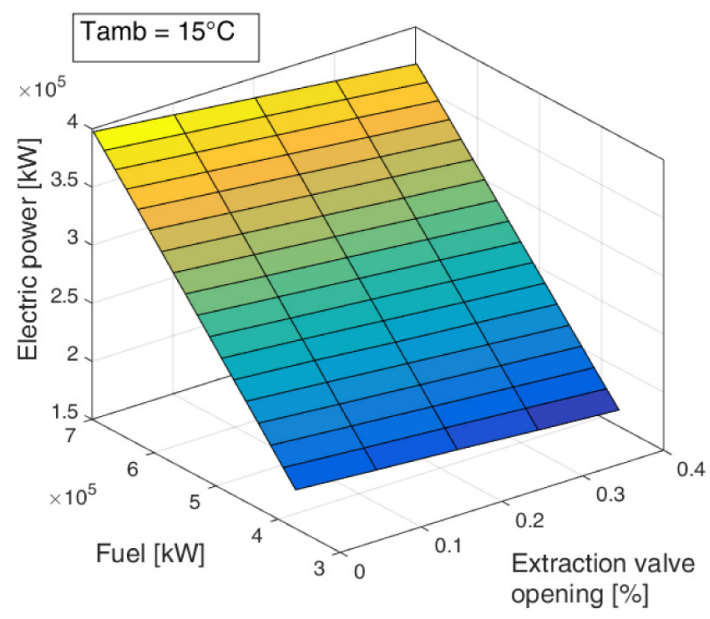

(a)

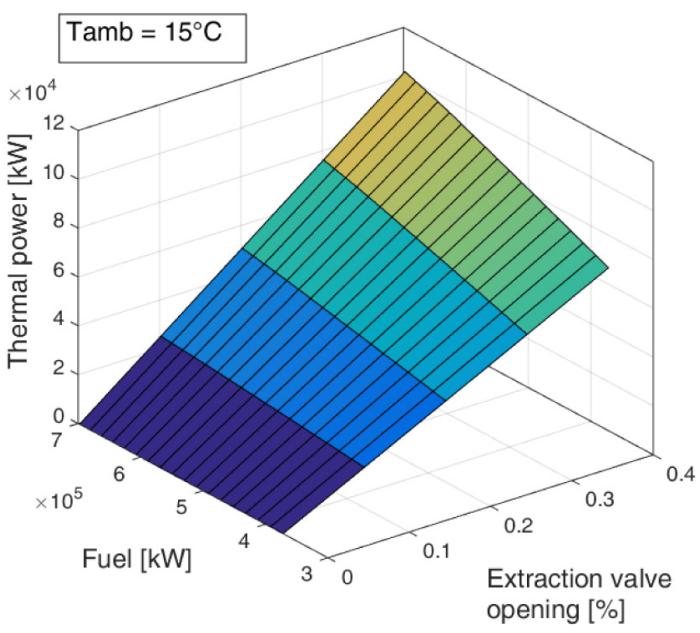

(b)

Fig. 4. Electric (a) and thermal (b) power outputs as functions of fuel and temperature for a CHP natural gas combined cycle with extraction-type steam turbine at fixed ambient temperature $15^{\circ} \mathrm{C}$.

The scheduling problem is still a very challenging MINLP (due to the nonlinearity of the performance curves of the units) however, as shown in Bischi et al. [10] and Dvořák et al. [46], it can be accurately linearized and converted into a MILP.

In this work, the approach and MILP model proposed by Bischi et al. [10] is considered and adapted. In particular, first the performance map of each unit is approximated by means of a datadriven approach considering performance curves either derived from experimental data or provided by the manufacturer. Then, the curves of one-degree-of-freedom units are approxi-mated with one-dimensional piecewise linear functions, while curves of twodegree-of-freedom units are approximated with two-dimensional piecewise linear functions and the triangle method [47]. The interested reader can find full details in Bischi et al. [10].

As an example, the performance curves of the commercially available Siemens SGT-100 gas turbine (with heat recovery) and of a CHP natural gas combined cycle based on the GE GT 9F.03 with extraction-type steam turbine are reported respectively in Figs. 3 and 4. The performance curves of the GT and NGCC have been determined by simulating the part-load behavior of the cycles with 
the commercial software GT PRO [48].

It is worth noting that for the CHP turbine considered in Fig. 3, the part-load performance map of the thermal power with respect to the fuel input (load) is nonlinear and concave while the one relative to the electric power is nonlinear and convex. Instead, the CHP NGCC considered in Fig. 4 has an almost linear part-load performance map relative to the electric power output but a nonlinear (neither convex nor concave) part-load map relative to the thermal power. It is important to recall that, since the optimization naturally aims at maximizing the unit power output for each given fuel input rate, having concave performance maps would allow to imple-ment the piecewise linear approximation without the need of introducing binary auxiliary variables associated to the intervals of the approximation [47]. As shown in Figs. 3 and 4, in this problem we have also nonconcave performance maps, making it necessary to use the general version of the piecewise linear approximation with binary auxiliary variables. In partic-ular, the well-known one-dimensional PWL approximation has been adopted for approximating the partload performance curves of the units with a single control variable (e.g., CHP GTs, CHP NGCCs with back-pressure turbine, and boilers), and the triangle method [47] has been selected for the twodimensional piecewise linear approximation of the part-load maps of the units with two control variables (i.e., the CHP GT with post firing and the CHP NGCC with extraction-type steam turbine). According to the computational results of Bischi et al. [10], these piecewise linear (PWL) approximations can yield accurate estimates of the optimal objective function values already with 10 interval approximations, even for non-smooth performance maps.
On the other hand, the computational time required to obtain accurate scheduling solutions may not be negligible even with state-of-the-art MILP solvers. Computational results [10] show that, when considering 24 time intervals (one operating day with an hourly basis discretization) and using CPLEX as MILP solver [22], up to 5 min may be necessary to find accurate solutions (relative MILP gap of 9E-3\%). Dealing in this work with a design problem and not a short-term scheduling problem, we have the following two objectives:

1. determine of the optimal scheduling throughout the whole operating time horizon (e.g., one year) so as to assess the actual part-load performance, operating costs and scheduling limitations of the selected units;

2. limit the computational time of the scheduling problem to a few tens of seconds because it needs to be solved for each design solution fixed by the upper level optimization algorithm.

The two objectives are conflicting because the longer is the time horizon, the larger is the number of time intervals, and the larger is the number of variables and constraints of the MILP. For instance, as reported in Ref. [5], for a relative gap of $0.1 \%$, the optimization of the daily operation problem (24 time intervals) with the MILP model proposed in Bischi et al. [10] takes tens of seconds while that of the weekly operation problem $(24 \times 7$ time intervals) may take even thousands of seconds. Extending the time horizon to the whole expected operating time horizon is clearly impracticable.

For this reason, the following assumptions, approximations and expedients must be introduced so as to limit the computational time required to solve the lower level scheduling problem:

(a)
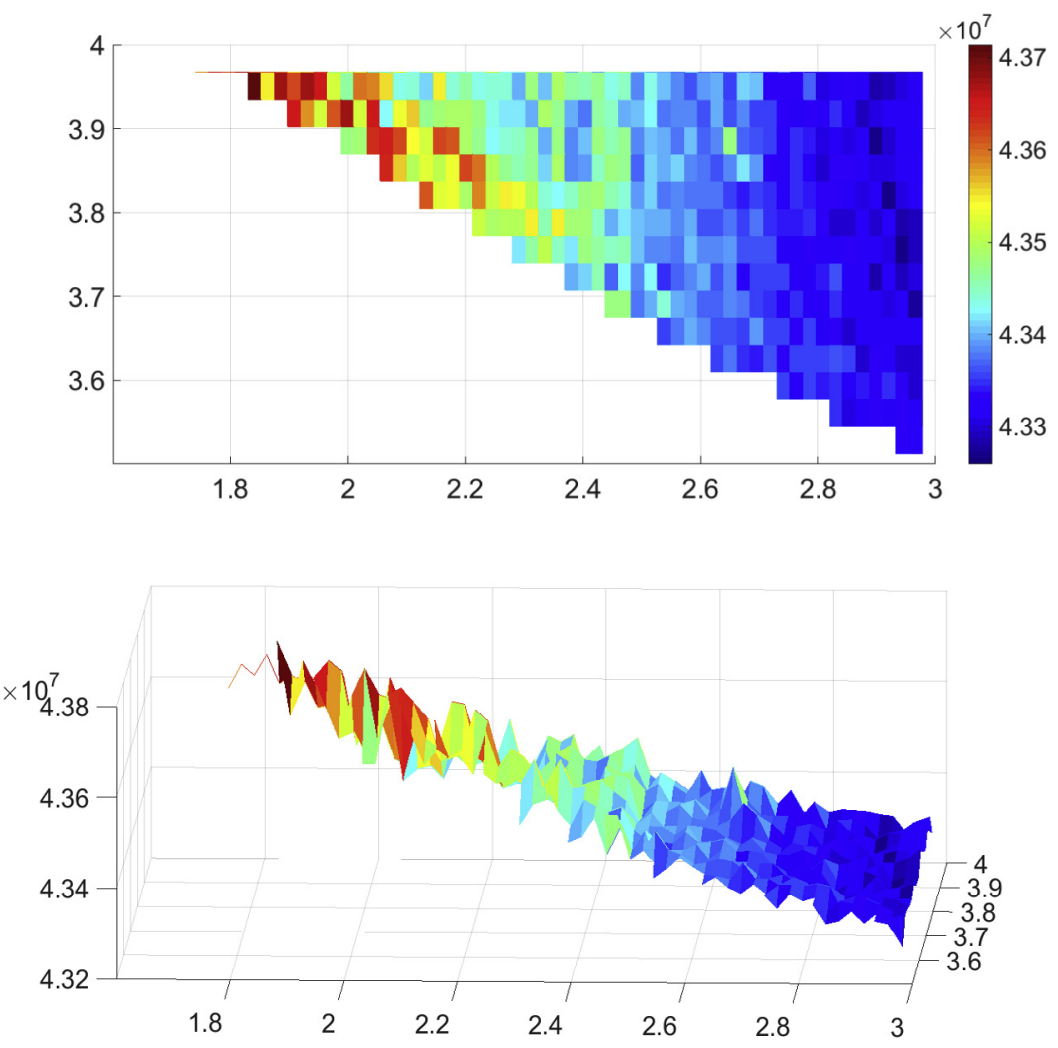

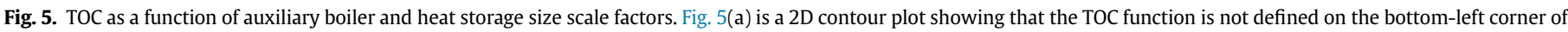
the box because the scheduling problem is infeasible. Fig. 5(b) is a 3D plot and it clearly shows that the objective function is non-smooth. 
1) First, rather than tackling the whole operating time horizon (e.g., year) at once within a single large scale MILP, it is more advantageous to decompose it into operating horizons of limited length (days or weeks) corresponding to simpler MILPs. However, since the system can potentially include a heat storage, it is important to consider reasonably long time horizons to fully exploit the storage system. For the heat storage systems typically used in in-dustrial and residential applications, the time horizon of one week appears to be a reasonable compromise between computational complexity and solution quality. Be-sides, the weekly horizon has the advantage of coinciding with the typical periodicity of the heat demand profile and electricity prices. For these reasons, we decided to consider weeks rather than days to represent the expected operating year. As a consequence, the assumption we make is that, if installed, the heat storage system level has a weekly periodicity (the tank levels at the beginning and end of the weeks are equal).

2) To limit the computational time, it is not worth considering and optimizing the scheduling of the 52 weeks of the year but it is necessary to determine a limited number (e.g., three) of most representative weeks. To this end, the clustering algorithm described in Section 5 has been developed. The approximation caused by the adoption of the representative weeks is limited to $3 \%$ (in terms of total operating costs of the whole operating year), as reported in Section 6.

3) Since we are not dealing with short-term scheduling problems but with a design problem, it is not essential to find very accurate scheduling solutions by solving the MILP to optimality with very small relative gaps as done in Bischi et al. [10]. Indeed, if a relative MILP gap of the order of $1 \%$ is adopted, the returned solution has a close-to-optimal objective function value (at most $1 \%$ higher than the global optimum) and its scheduling is very close to the optimal one while the average computational time may be decreased by a factor of $5-10$ (from $10-100 \mathrm{~s}$ to $1-20 \mathrm{~s}$ ).

4) As an expedient to further decrease the computational time of the lower level problem without introducing additional approximations or assumptions, an adaptive bounding procedure is adopted. The basic idea is to begin with a quick and rough estimate of the scheduling of the representative weeks with a large MILP relative gap (e.g., 10\%), evaluate the lower bound of the TAC of the solution, and then improve the scheduling solution accuracy considering the relative gap of $1 \%$ only if the solution is actually promising compared to the best-known solution. A detailed description of this bounding procedure is reported in Subsection 4.3.

\subsection{Upper level problem}

The upper level optimization problem explores the set of possible CHP system configurations and designs (sizes), in continuous interaction with the lower level problem as the operating performance of each design solution is evaluated by solving the lower level problem.

It is important to notice that the corresponding MINLP problem may be non-smooth (non-differentiable and discontinuous) with respect to the continuous design variables (unit sizes) since the value of the objective function (total annual cost) depends on the scheduling solution of the lower level MILP. Indeed, an infinitesimal variation of the units' sizes may lead to a finite change of the TOC, lower level objective function, because a different combination of integer variables become optimal. The non-smoothness of the TOC function is then transferred to the TAC function minimized at the upper level. As an example, a 2D contour plot and a 3D plot of the TOC as a function of the sizes of two (co)generation units are reported in Fig. 5.

Moreover, the TOC function is affected by numerical noise due to the tolerance (relative MILP gap) of the MILP solver. Furthermore, its value may not be defined in some points of the design variables feasible region because the lower level MILP solver may fail to find a feasible scheduling solution (see Fig. 5). For these reasons, the upper level MINLP problem is particularly challenging as gradient-based branch-and-bound methods (like e.g., BARON [49]) are not suitable and robust heuristic optimization algorithms must be used. Due to the slow convergence rate of the optimization algorithm (penalized by the lack of information from the knowledge of the function gradient), the problem formulation must be carefully adapted so as to limit the number of variables. For this reason, three different formulations of the upper level problem are derived. For each formulation, specific evolutionary optimization algorithms are adopted and the obtained results are compared.

1. Strategy 1. The basic idea is to formulate the upper-level problem as a MINLP with binary, discrete and real variables and tackle it with the evolutionary algorithm eSS [50].

2. Strategy 2. It is a partially relaxed version of the MINLP problem where the discrete size variables $y_{\mathrm{D}, i}$ are removed by assuming that all the units have continuous sizes (also those available only in discrete sizes). A binary variable is defined for the selection of each unit and a continuous variable for its size (for all machines in sets $C U$ and $C A$ ). Then, a heuristic procedure is used to select the discrete size, closest to the relaxed solution, which preserves solution feasibility. The problem is tackled with the evolutionary algorithm eSS [50].

3. Strategy 3. The MINLP problem is converted into a discontinuous Non-Linear Problem (NLP) where the all the discrete/binary variables are removed and only sizes of the units are optimized as real variables. All the units may have continuous sizes ranging from zero to the maximum available one. If the size is lower than the minimum available size, an "if" condition deactivates the units (i.e., it means that the unit is not selected). The problem is tackled with the derivativefree method PGS-COM [51] specifically developed to deal with non-smooth discontinuous functions.

\subsubsection{Strategy 1}

A binary variable $z_{\mathrm{D}, i}$ and a real variable $x_{\mathrm{D}, i}$ are defined for the selection and the size of the units with custom size (those in set $C U$ ), and a discrete variable $y_{\mathrm{D}, i}$ is defined for the selection of the units with discrete size (those in $C A$ ). If $y_{\mathrm{D}, i}=0$, the $i$-th unit type (e.g., gas turbine) is not selected, while if $y_{\mathrm{D}, i}=Y_{i, k}$, the unit is selected with the commercially available size $Y_{i, k}$. If it may be worth installing multiple units of the same type and size, multiple discrete variables $y_{\mathrm{D}, i}$ must be defined (one for each potential unit). Compared to Problem (P2), this formulation allows to avoid defining binary variables for the selection of units with discrete size. The formulation of the upper level problem is reported in Problem (P3). 


$$
\min _{z_{\mathrm{D}, i}, x_{\mathrm{D}, i}, y_{\mathrm{D}, i}, l_{\mathrm{H}, \mathrm{storage}}} T A C
$$

s.t.

$g_{\mathrm{D}}\left(z_{\mathrm{D}, i}, x_{\mathrm{D}, i}, y_{\mathrm{D}, i}\right) \leq 0$

$X_{\mathrm{D} \min , i} \leq x_{\mathrm{D}, i} \leq X_{\mathrm{D} \max , i} \quad x_{\mathrm{D}, i} \in \mathbb{R} \quad \forall i \in C U$

$y_{\mathrm{D}, i} \in\left\{\mathrm{Y}_{i, 1}, Y_{i, 2}, Y_{i, 3}, \ldots\right\} \quad \forall i \in C A$

$l_{\mathrm{H}, \text { storage,min }} \leq l_{\mathrm{H}, \text { storage }} \leq l_{\mathrm{H}, \text { storage,max }} l_{\mathrm{H}, \text { storage }} \in \mathbb{R}$

$z_{\mathrm{D}, i} \in\{0,1\} \quad \forall i \in C U$

It is worth noting that the evaluation of the objective function actually implies the solution of the lower level MILP scheduling/ operational problem.

Problem (P3) is a "black-box" MINLP problem and it is tackled with the evolutionary algorithm eSS [50] which can handle both discrete and continuous variables, as well as non-smooth objective functions, as reported in Refs. [50] and [51]. eSS is a global optimization algorithm for MINLPs based on a combination of two meta-heuristic methods, scatter search [52] and path-relinking [53]. The eSS algorithm starts from a population of $N$ solutions, half obtained by the latin hypercube sampling and half by random sampling. Then, every iteration consists of four main steps: (i) a hyper-rectangle based combination procedure which generates $\mathrm{N}-1$ new solutions (offspring) for every population member (parent) according to a heuristic search strategy aimed at generating more solutions close to the best members, (ii) a procedure to explore promising directions, called "go-beyond" strategy, (iii) a population update step which replaces the parent solution with the best offspring solution, if the latter outperforms the former, (iv) a population check step to replace individuals stuck in a local optima and to replace them with randomly generated solutions. Further details about the eSS algorithm can be found in Ref. [50]. The maximum number of function evaluations is used in this work as stopping criterion. As far as the parameter values of the algorithm are concerned, in this work we have adopted the same values recommended by the authors in the user's guide for non-smooth functions.

\subsubsection{Strategy 2}

It is a slightly relaxed version of the MINLP problem where the discrete variables are converted into continuous ones by assuming that the units in $C A$ are available in a continuous range of sizes. A binary variable is defined for the selection of each unit and a continuous variable for the sizes. As size is continuously varied, its effect on the specific capital cost and performance of the units is modelled with the scale-law equation, as in Ref. [16]. The problem formulation is reported in Problem (P4). $\min _{z_{\mathrm{D}, i}, x_{\mathrm{D}, i}, \mathrm{JD}_{\mathrm{D}, i}, l_{\mathrm{H}, \text { storage }}} T A C$

s.t.

$g_{\mathrm{D}}\left(z_{\mathrm{D}, i}, x_{\mathrm{D}, i}, y_{\mathrm{D}, i}\right) \leq 0$

$X_{\mathrm{D} \min , i} \leq x_{\mathrm{D}, i} \leq X_{\mathrm{D} \max , i} \quad x_{\mathrm{D}, i} \in \mathbb{R} \quad \forall i \in C U$

$Y_{\mathrm{D} \min , i} \leq y_{\mathrm{D}, i} \leq Y_{\mathrm{D}_{\max , i}} y_{\mathrm{D}, i} \in \mathbb{R} \quad \forall i \in C A$

$l_{\mathrm{H}, \text { storagemin }} \leq l_{\mathrm{H} \text {,storage }} \leq l_{\mathrm{H}, \text { storage,max }} l_{\mathrm{H}, \text { storage }} \in \mathbb{R}$

$z_{\mathrm{D}, i} \in\{0,1\} \quad \forall i \in A l l$ 
Problem (P4) is still a "black-box" MINLP (the evaluation of the objective function actually implies the solution of the lower level MILP scheduling/operational problem) but with a lower number of integer variables compared to the original one. After the eSS algorithm finds the best solution of Problem (P4), the values of the relaxed continuous variables are converted back to the closest discrete values with a heuristic procedure. The increase/decrease of the nominal thermal power due to the discretization of the sizes of units must be compensated subtracting/adding the difference to auxiliary boilers. The final objective function value of the discrete set of variables is then calculated in the black-box.

\subsubsection{Strategy 3}

It is a relaxed version of the upper level synthesis/design problem where all the binary and discrete variables are removed. The discrete variables related to the selection of the unit size are converted into continuous ones by assuming that the units in $C A$ are available in a continuous range of sizes. The size effect on the specific capital cost and performance of the units is modelled with the scale-law equation, as in Ref. [16]. The binary variables for the selection of the units have been replaced by an "if" statement included in the black-box which evaluates the objective function by computing the capital costs of the units and solving the lower level MILP scheduling problem. The "if" statement considers as "selected" the units with size larger than the minimum one. If the size of the unit is lower than the minimum available size (i.e., if $X_{\mathrm{D}}$ $\min , i>x_{\mathrm{D}, i}$ or $Y_{\mathrm{D} \min , i}>y_{\mathrm{D}, i}$, the "if" condition considers than the unit has not been selected. The problem can be formulated as re-ported below.

$$
\min _{x \mathrm{D}, i, y \mathrm{D}, l_{\mathrm{H}, \mathrm{storage}}} T A C
$$

s.t.

$g_{\mathrm{D}}\left(x_{\mathrm{D}, i}, y_{\mathrm{D}, i}\right) \leq 0$

$X_{\mathrm{D} \min , i} \leq x_{\mathrm{D}, i} \leq X_{\mathrm{D} \max , i} \quad x_{\mathrm{D}, i} \in \mathbb{R} \quad \forall i \in C U$

$Y_{\mathrm{D} \min , i} \leq y_{\mathrm{D}, i} \leq Y_{\mathrm{D} \max , i} \quad y_{\mathrm{D}, i} \in \mathbb{R} \quad \forall i \in C A$

$l_{\mathrm{H}, \text { storagemin }} \leq l_{\mathrm{H}, \text { storage }} \leq l_{\mathrm{H}, \text { storage,max }} l_{\mathrm{H} \text {,storage }} \in \mathbb{R}$

Since all variables are continuous, Problem (P5) is a black-box constrained Non-Linear Problem (NLP). The objective function is extremely discontinuous because, in addition to the discontinuity points due to the lower level MILP, the "if" statement within the black-box generates step-type discontinuity on the points corresponding to the minimum size of the units $\left(X_{\mathrm{D} \min , i}, Y_{\mathrm{D} \min , i}\right)$. The problem is tackled with the derivative-free method PGS-COM [51] specifically developed to deal with discontinuous functions.

The PGS-COM algorithm combines the positive features of the Constrained Particle Swarm Optimizer (CPSO) of Hu and Eberhart (2002) [54], Generating Set Search of Lewis et al. (2007) [55], and the Complex of Andersson (2001) [56]. Each iteration of the algorithm consists of three steps: (i) a search step corresponding to a population update of a revised CPSO, (ii) an optional (skipped if the CPSO improves the best solution found so far) poll step corresponding to an iteration of the GSS around the best solution found so far, and (iii) a few optional (skipped if either the CPSO or the GSS step finds a better solution) reflection steps corresponding to a few iterations of the Complex algorithm. The algorithm stops when the swarm size (defined as the maximum distance between the best particle and the remaining particles), the step size parameter of the GSS step and the size of the population used by the Complex step become smaller than given threshold or the maximum number of function evaluations is reached. The computational results presented in Ref. [51] indicate that for noisy non-smooth black-box problems PGS-COM performs better than eleven ad-hoc methods. PGS-COM has been successfully applied to the optimization of a large number of energy systems, such as heat recovery steam cycles [57], $\mathrm{CO}_{2}$ capture processes [58] and Organic Rankine Cycles [59]. As far as the parameter values of the algorithm are concerned (e.g., number of swarm particles, neighborhood size, minimum GSS step size parameter, etc.), in this work we have adopted the same values recommended by the authors in Ref. [51].

\subsection{Two-stage algorithm and bounding procedure scheme}

The block-flow diagram of the overall algorithm is reported in Fig. 6.

The upper-level algorithm (eSS in strategy 1 and 2 or PGS-COM in strategy 3 ) determines, on the basis of its search strategy, the

$$
\}
$$

most promising system design solutions $\left(z_{\mathrm{D}, i}, x_{\mathrm{D}, i}, y_{\mathrm{D}, i}, l_{\mathrm{H}, \text { storage }}\right)$ to evaluate. For fixed system design solutions, the lower-level MILP scheduling optimization problem is solved by the CPLEX branchand-bound algorithm [22] for a certain set of typical weeks (see Section 5). Typical weeks are optimized independently so as to limit the size of the MILP and then the computational time. The solution of the scheduling problem for each typical week allows to estimate the operating costs and revenue of the whole operating time horizon (one year). Since the computational time required to solve the MILP with a relative MILP gap equal to $1 \%$ (yielding a good compromise between solution accuracy and computational time) is 


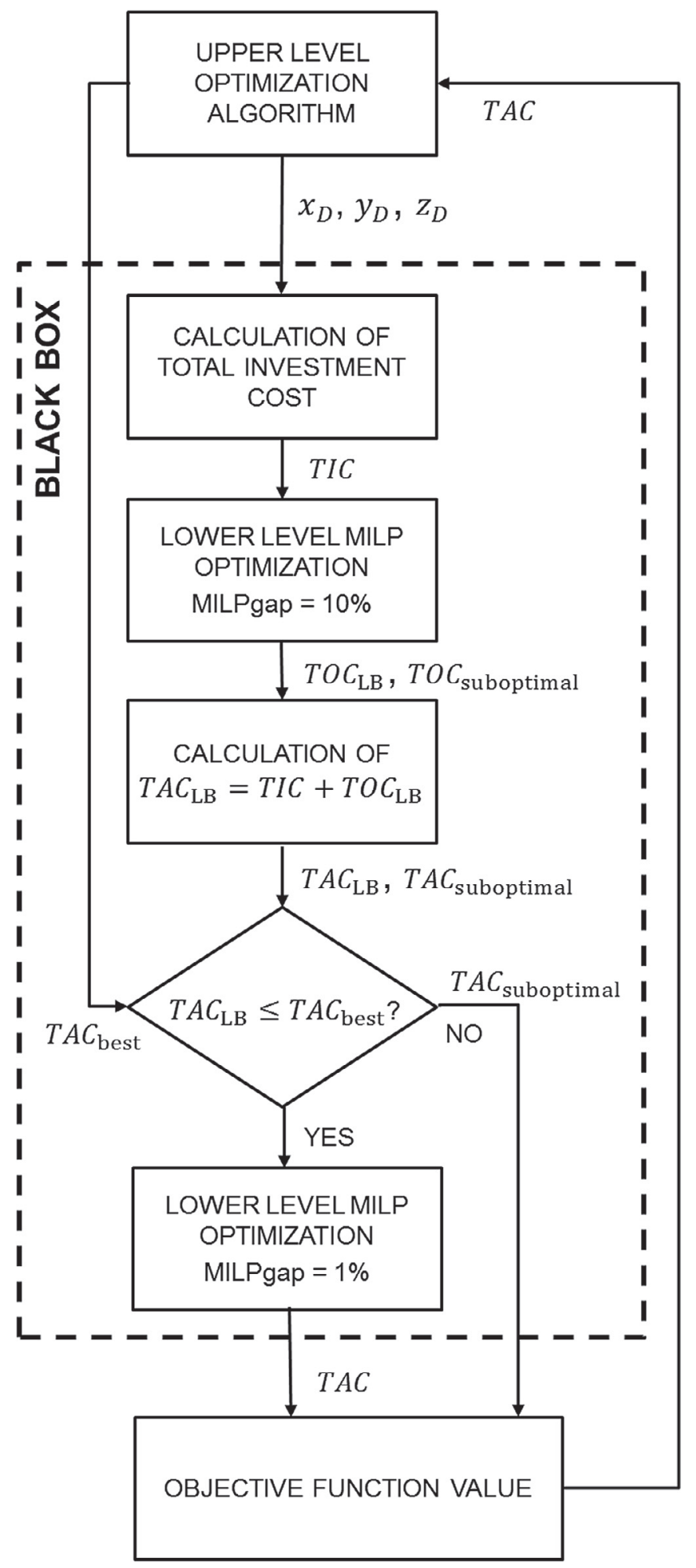

Fig. 6. Block-flow diagram of the devised two-stage algorithm with bounding procedure.

of the order of 10-100 s and black-box algorithms require several thousands of function evaluations to find close-to-optimal solutions (as shown in Ref. [51]), the computational time required to complete an optimization would be even longer than $72 \mathrm{~h}$. As an expedient to decrease the computational time without introducing additional approximations or assumptions, a bounding criterion is

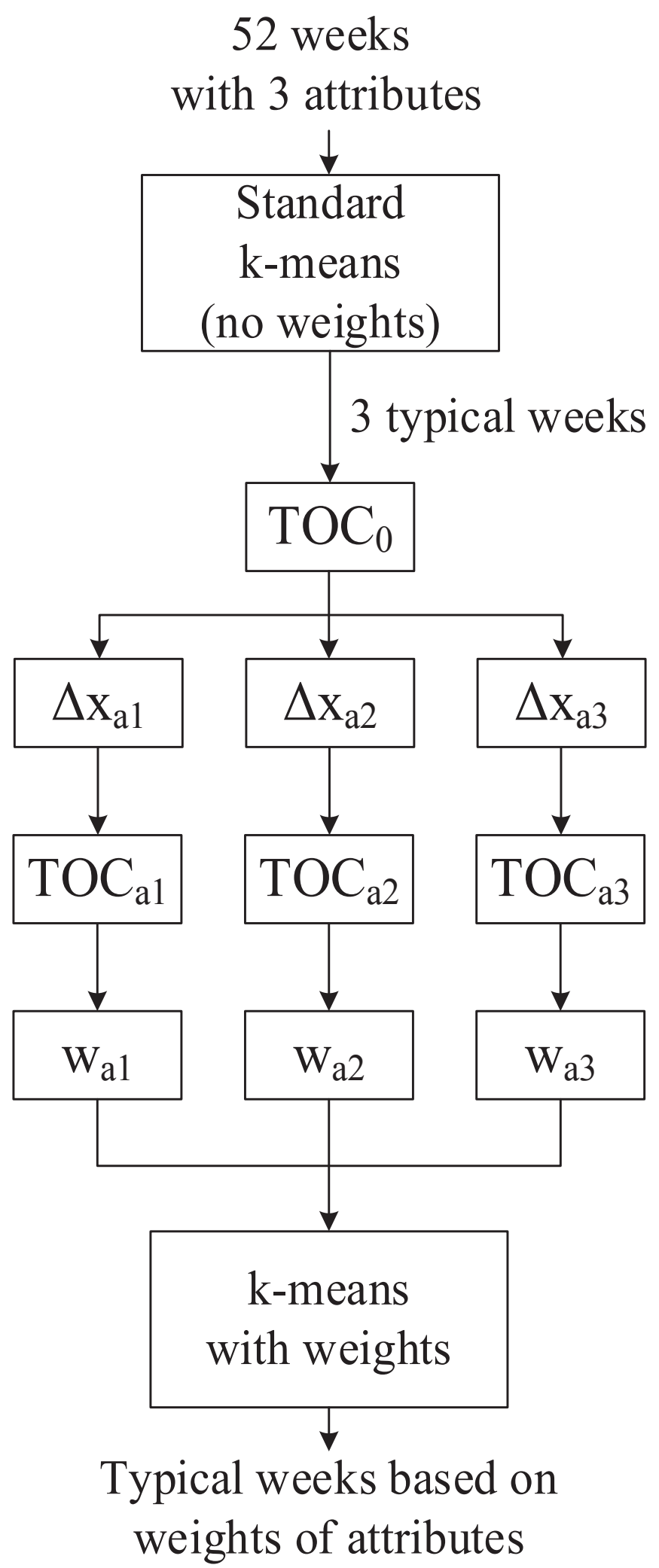

Fig. 7. Block-flow diagram of the weighted clustering k-means algorithm.

developed based on the property of the "lower bound" of the objective function value computed and returned by the MILP branch-and-bound algorithm $\left(T O C_{\mathrm{LB}}\right)$. Integer programming theory [21] guarantees that the objective function of the optimal solution 
of the MILP (TOC) is greater than or at most equal to such lower bound. In particular, the lower bound is determined by fixing the value of the integer/binary variable which is subject to the "branching" operation, and considering the remaining integer variables as continuous variables. For each branch (i.e, possible value of the integer variables which is subject to branching), the corresponding "relaxed" (continuous) linear optimization problem is efficiently solved with linear programming algorithms. Among the branches, the best solution of the relaxed optimization problems is certainly better than (or at most equal to) any integer solution of the MILP. For this reason, it can be considered as a lower bound of the minimum of the MILP problem. As the branch-andbound algorithm proceeds exploring more branches, less integer variables are relaxed because their optimal value is known and fixed. As a consequence, across the iterations the lower bound tends to increase while the objective function value of the best found integer solution decreases. The relative MILP gap is the relative difference between the lower bound and the best found integer solution and it is the convergence criterion of the branchand-bound algorithm.

Since it was observed that for the scheduling MILP problem the computational time to reach a MILP gap of $10 \%$ is on average less than $1-20$ s and a steep increase occurs below that threshold value, a significant saving of computational time can be achieved by adopting a large MILP gap for most of the sampled design solutions and adopting a small MILP gap (1\%) only for those which have the potential to improve the best current solution. Indeed it is not worth performing an accurate optimization of the scheduling of the system designs which are certainly worse than the best current solution. The idea, which is at the basis of the following procedure, is to use the lower bound of the MILP scheduling problem $\left(T O C_{\mathrm{LB}}\right)$ to assess whether a design solution has the potential to improve the best current solution or not:

(i) Perform a quick and fairly accurate estimate of the scheduling of each design solution for the selected typical weeks adopting a large MILP relative gap (e.g., 10\%),

(ii) Read the lower bound of the total operating cost $\left(T O C_{\mathrm{LB}}\right)$ of the solution, and then compute the corresponding lower bound of total annual cost $\left(T A C_{\mathrm{LB}}\right)$ as the actual investment cost of the system plus $T O C_{\mathrm{LB}}$.

(iii) If the design solution has a $T A C_{\mathrm{LB}}$ better (lower) than the best-known solution found so far (then it is a promising solution because its actual TAC may be lower than that of the best-known solution found so far), the accuracy of the scheduling solution is improved by solving the MILP with a smaller relative gap (e.g., 1\%). Instead, if the design solution has a $T A C_{\mathrm{LB}}$ higher than the best-known solution found so far it is not worth improving the accuracy of its scheduling.

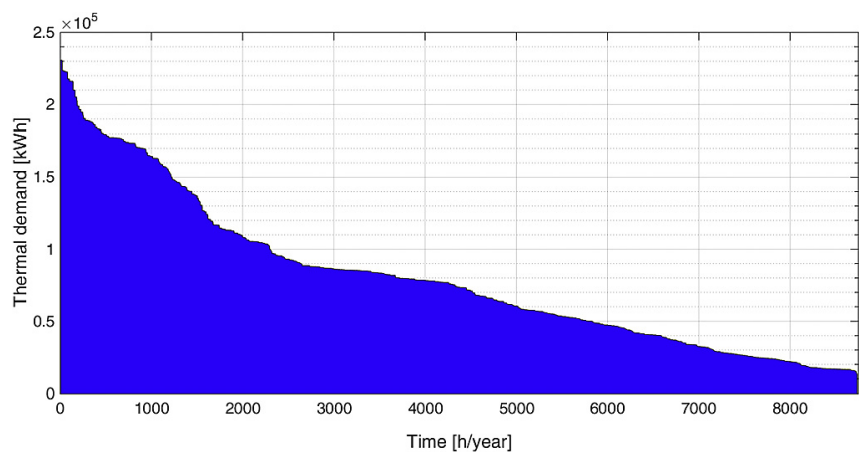

Fig. 8. Heat load duration curve of the district heating network.
For the test cases presented in Sections 6 and 7, the optimization runs (solution of both lower level and upper level problems) adopting the above-described bounding procedure allows to save about $30 \%$ of computational time (e.g., about $22 \mathrm{~h}$ over a total of $76 \mathrm{~h}$ for test case B described in Section 7) with respect to runs in which the procedure is not used (i.e., the scheduling of each sampled designs is optimized with the MILP gap of 1\%).

In summary, the proposed two-stage algorithm has the following advantages:

1. The upper level problem features a relatively small number of variables (in the range 20-30 for the industrial scale problems) and this decreases the probability of converging to local minima.

2. It is possible to handle any non-linear relation, even discontinuous, between performance and unit size as the upper level optimizers can handle discontinuous functions.

3. It is possible to consider multiple and longer typical operating periods, such as several typical weeks, without incurring in excessively challenging scheduling problems. This is due to the possibility of solving each typical week independently.

4. The two-level decomposition does not cause any approximation compared to the original MINLP problem. Very minor approximations are introduced only by the piecewise linear linearization of the performance curves of the units necessary to convert lower level (scheduling) problem into a MILP.

5. Thanks to the procedure based on the lower bound of the lower level MILP problem, the computational time required to reach convergence does not exceed $55 \mathrm{~h}$, even without parallel computing on a standard workstation.

6. The design solutions sampled by the upper-level evolutionary algorithm (eSS and PGS-COM) could be evaluated in parallel on the available processors of the workstation, with an almost proportional decrease of computational time.

On the other hand, the solution quality of the design solutions found the by the upper level algorithms (eSS or PGS-COM) cannot be guaranteed as the algorithms are heuristic. Similarly to other evolutionary algorithms, it is recommended to repeat each optimization at least 10 times with randomized starting solutions, as done in this work (see Section 7).

\section{Selection of typical weeks}

The use of a set of typical periods for representing a year provides an efficient method for decreasing the number of data points, and consequently the number of parameters, variables and constraints of the multi-period optimization model. We assume that a year can be approximated by a smaller set of representative periods. As written in Section 4.1, rather than tackling the whole operating time horizon (i.e., year) at once within a single large scale MILP, it is more advantageous to decompose it into weekly operating horizons with an hourly time discretization. Indeed, the week yields a good compromise between the computational complexity of the MILP scheduling problem and the possibility of exploiting the heat storage system (as heat demand profiles and electricity prices typically have a weekly periodicity). To limit the computational time required to solve the scheduling problems, it is not worth considering and optimizing the scheduling of the 52 weeks of the year. Bischi et al. [5] utilized a simplified rolling horizon approach in order to avoid the resolution of the yearly problem at once, they solved all the 52 weeks of the year separately while keeping into account yearly constraints via several loops. Such approach which was considered to be more accurate, especially in that case whether they had to keep into account monthly and yearly constraints depending on fiscal incentives, but it takes up to one day of time for 
a yearly instance. In order to save computational time and because we do not have to keep into account constraints binding time periods larger than a week, we decided to determine a limited number of weeks representative of the whole year. The problem of the selection of typical weeks can be stated as following.

Given:

- the expected hourly profiles of heat and electricity demands over a time horizon of one year (52 weeks);

- the expected hourly profiles of ambient temperature of the installation site over a time horizon of one year (52 weeks),

determine a set of 3 representative weekly periods, and in particular:

- partition the 52 original weeks in 3 clusters, each represented by one typical week;

- determine the representative hourly profiles of heat and electricity demands of each typical week;

- determine the representative hourly profiles of ambient temperature of the installation site of each typical week,

so as to minimize the overall within-cluster errors (i.e., the sum of the errors between each original week and its typical week in all clusters).

To this end, the k-means clustering algorithm [60] with repetitions has been adopted.

Compared to previous works (Fazlollahi et al., 2014 [11], Dominguez-Munoz et al., 2011 [61]) which used the plain kmeans algorithm to determine the typical periods, we assign different weights to the relative errors of ambient temperature, electricity and heat. Indeed, when determining the demand profiles of the typical operating periods, it is not correct to consider the electricity demand and the heat demand with the same relevance. While electricity can be imported/exported from/to the electric grid and then the user's demand of electricity is not constraining the scheduling of the CHP units, heat has to be generated by installed CHP units so the user's demand of heat is actually constraining the scheduling of the system. Another example is the ambient temperature profile which may be considered among the input data because of its influence on the performance of the CHP units (Bischi et al., 2014 [10]). However the effect of ambient temperature on the performance of the system is expected to be much lower than that of electricity and heat demands.

A possible approach to determine the relative weights of the electricity demand profile, heat demand profile and ambient temperature on the scheduling of CHP units is proposed in the following lines and illustrated in Fig. 7.
Table 2

Weights for the attributes in the extreme cases of the design problem.

\begin{tabular}{llll}
\hline & $\begin{array}{l}\text { Electricity } \\
\text { demand }\end{array}$ & Heat demand & $\begin{array}{l}\text { Ambient } \\
\text { temperature }\end{array}$ \\
\hline GT-SC and storage & 0.3686 & 0.6934 & 0.0309 \\
GT-PF and storage & 0.3914 & 0.6631 & 0.0281 \\
CC-BP and storage & 0.3727 & 1.0667 & 0.0353 \\
CC-BE and storage & 0.8642 & 1.2237 & 0.0938 \\
AB only & 0.4363 & 0.7607 & 0.0 \\
\hline
\end{tabular}

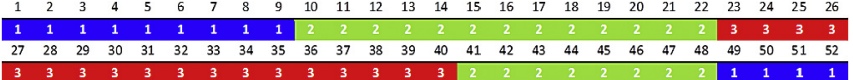

Fig. 9. Assignments for the test case with the weighted k-means method for the design problem. Blue denotes typical week 1, green denotes typical week 2 and red typical week 3. (For interpretation of the references to colour in this figure legend, the reader is referred to the web version of this article.)

1. The k-means algorithm is applied to determine a first estimate of the typical weeks with equal (unitary) weights given to the errors of electricity demand, heat demand and ambient temperature. Then the optimal scheduling for the set of typical weeks is computed and the value of the objective function $T O C_{0}$ is obtained.

2. For every attribute $a$ (ambient temperature, heat demand, electricity demand), one by one, the corresponding data are shifted by a fixed value $\Delta x_{a}$ that represent a percentage of the average value of the attribute $a$ of the entire original dataset (e.g., if the average heat demand is $100 \mathrm{MWh}$, a $10 \mathrm{MWh}$ heat demand is summed to each operating hour of the year). For instance, $\Delta x_{a}=10 \%$ is a reasonable value as it does not cause a considerable alteration of the original profile while allowing to appreciate the effect of the attribute $a$ on the system performance. Each attribute is in this way associated to a "shifted profile".

3. One by one, the "shifted profiles" are used as basis to generate "shifted typical weeks" (one shifted typical week associated to each shifted profile). The optimal scheduling of the shifted typical weeks is then computed. If the scheduling problem for the shifted typical weeks results to be infeasible (e.g., undersized units), a smaller $\Delta x_{a}$ must be adopted in step $2 . T O C_{a}$ denotes the objective function value of shifted typical week associated to attribute $a$.

4. The weight of each attribute $w_{a}$ is then calculated as a finite difference: the ratio between the relative variation of the objective function values $T O C_{a}$ of the shifted typical weeks compared to the original typical weeks value $T O C_{0}$ and the relative shift of the profile for attribute $a$ :

Table 1

Available units for test cases A and B with their efficiency and economic data

\begin{tabular}{|c|c|c|c|c|}
\hline & Electric efficiency (full-load) & Specific capital cost (total installed costs) & $\begin{array}{l}\text { Number } \\
\text { of units }\end{array}$ & Available Sizes \\
\hline GT-SC & $\begin{array}{l}30 \% \text { (ref. size } 11 \mathrm{MW} \text {, scale factor on } \\
\text { heat rate } 0.90 \text { ) }\end{array}$ & $1300 \$ / \mathrm{kW}$ (ref. size $11 \mathrm{MW}_{\mathrm{el}}$, scale factor 0.78 ) & 4 & $\begin{array}{l}\text { Discrete: } 5042 \mathrm{~kW}_{\mathrm{el}} ; 6460 \mathrm{~kW}_{\mathrm{el}} ; 7784 \\
\mathrm{~kW}_{\mathrm{el}} ; 11176 \mathrm{~kW}_{\mathrm{el}}\end{array}$ \\
\hline GT-PF & $\begin{array}{l}30.5 \% \text { (ref. size } 16 \mathrm{MW} \text {, scale factor on } \\
\text { heat rate } 0.90 \text { ) }\end{array}$ & $1350 \$ / \mathrm{kW}$ (ref. size $16 \mathrm{MW}_{\mathrm{el}}$, scale factor 0.78 ) & 4 & $\begin{array}{l}\text { Discrete: } 16357 \mathrm{~kW} \text { el; } 21071 \mathrm{~kW}_{\mathrm{el}} \\
25516 \mathrm{~kW}_{\mathrm{el}} ; 27113 \mathrm{~kW}_{\mathrm{el}}\end{array}$ \\
\hline CC-BP & $\begin{array}{l}48.7 \% \text { (ref. size } 70 \mathrm{MW} \text {, scale factor on } \\
\text { heat rate } 0.95 \text { ) }\end{array}$ & $1430 \$ / \mathrm{kW}$ (ref. size $70 \mathrm{MW}_{\mathrm{el}}$, scale factor 0.81 ) & 4 & $\begin{array}{l}\text { Discrete: } 68254 \mathrm{~kW}_{\mathrm{el}} ; 70516 \mathrm{~kW}_{\mathrm{el}} \\
116692 \mathrm{~kW}_{\mathrm{el}} ; 136995 \mathrm{~kW}_{\mathrm{el}}\end{array}$ \\
\hline CC-BE & $\begin{array}{l}57.4 \% \text { (ref. size } 400 \mathrm{MW} \text {, scale factor on } \\
\text { heat rate } 0.95 \text { ) }\end{array}$ & $1030 \$ / \mathrm{kW}$ (ref. size $400 \mathrm{MW}_{\mathrm{el}}$, scale factor 0.81 ) & 4 & $\begin{array}{l}\text { Discrete: } 398195 \mathrm{~kW} \text { el } 413391 \mathrm{~kW} \text { el; } \\
435562 \mathrm{~kW}_{\mathrm{el}} ; 444416 \mathrm{~kW}_{\mathrm{el}}\end{array}$ \\
\hline $\mathrm{AB}$ & $\begin{array}{l}90 \% \text { (ref. size } 33 \mathrm{MW} \text {, scale factor on } \\
\text { heat rate } 0.99 \text { up to } 100 \mathrm{MW} \text { ) }\end{array}$ & $222 \$ / \mathrm{kW}$ (ref. size $33 \mathrm{MW}_{\mathrm{th}}$, scale factor 0.80) & 2 & Continuous: $10000-100000 \mathrm{~kW}_{\mathrm{th}}$ \\
\hline Storage & & & 1 & Continuous: $15000-80000 \mathrm{kWh}_{\mathrm{th}}$ \\
\hline
\end{tabular}



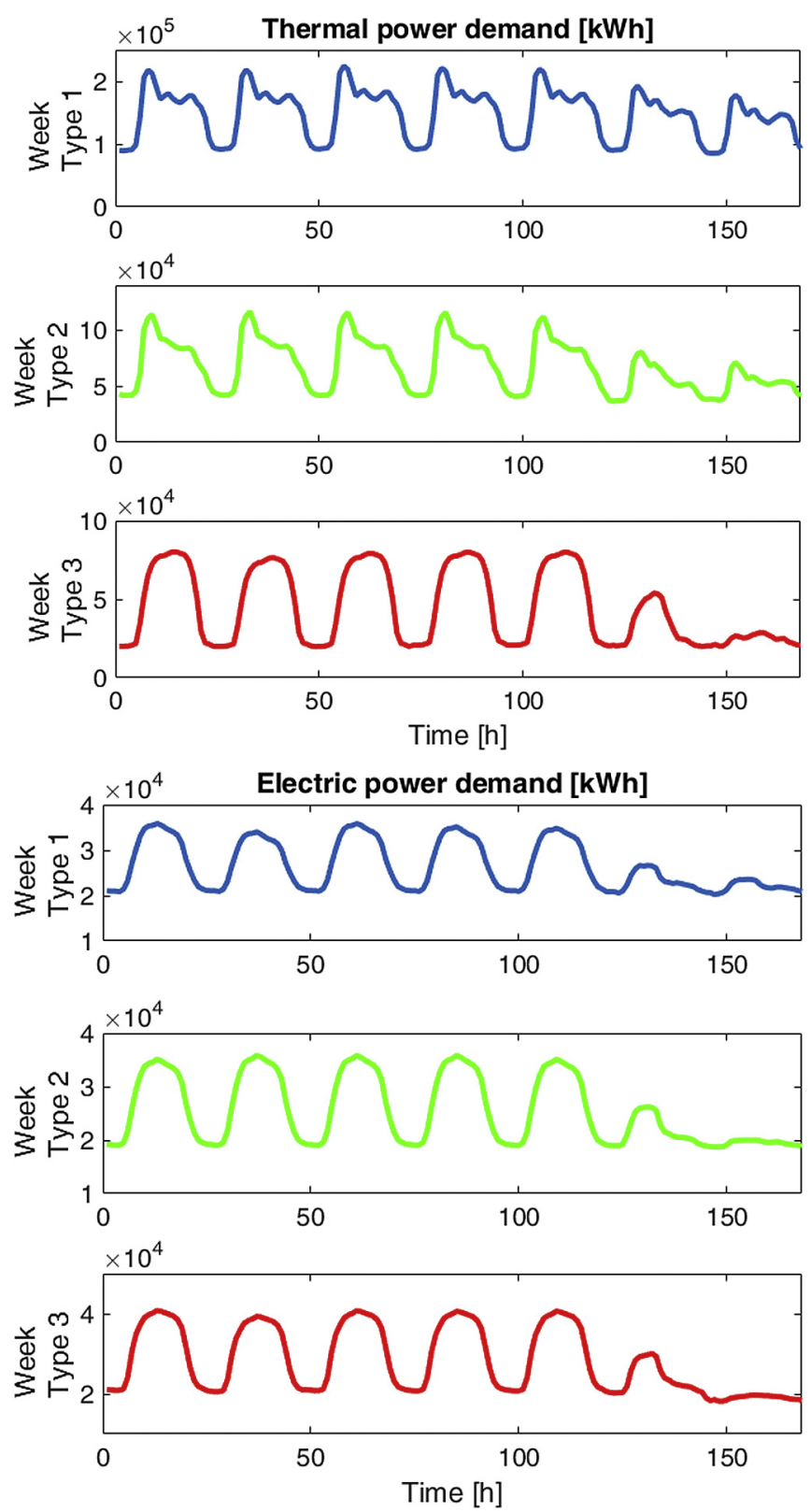

Fig. 10. Profiles of the thermal (a) and electric (b) power demands of the typical weeks. Blue denotes typical week 1 , green denotes typical week 2 and red typical week 3 . (For interpretation of the references to colour in this figure legend, the reader is referred to the web version of this article.)

$w_{a}=\frac{\frac{T_{O C}-C_{0} C_{a}}{T O C_{0}}}{\Delta x_{a}}$

After obtaining weights for all attributes, the weighted clustering k-means algorithm is able to form custom-fitted typical weeks for the corresponding problem.

Since the values of the weights may in general depend on the type (technology) and size of the installed CHP units, it is recommended to repeat the above-described procedure with different system designs (either for the most promising ones or for a set of extreme system configurations maximizing the use of each available technology, as done in the test case reported in Section 6) and then take the average values of the weights.

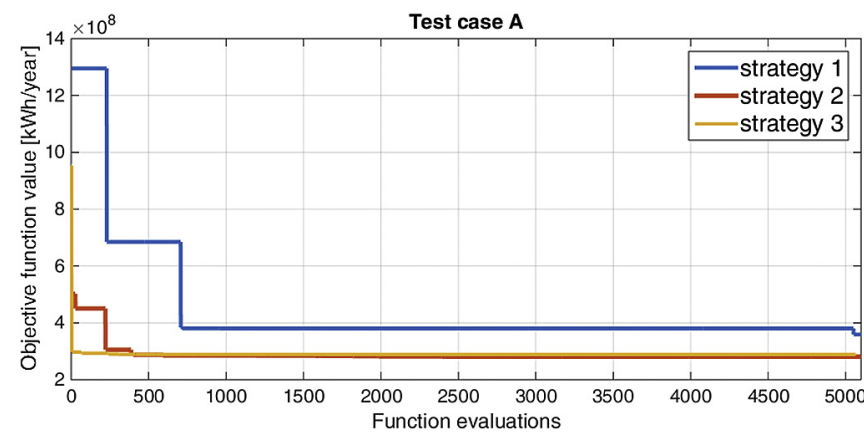

Fig. 11. Convergence plot (objective function value of the best found solution as a function of the number of function evaluations) of the three solution strategies on test case A.

\section{Test cases}

The presented optimization approach is applied to two test cases so as to compare the three strategies proposed in Subsection 4.2 and assess the quality of the solutions. The two test cases consist in the optimization of the design of a medium-size district heating network located in Northern Italy for two different objective functions, as further explained below. The network provides heat and electricity to residential buildings, offices, schools and com-mercial buildings. Electric and thermal demand profiles were derived from an accurate reconstruction of real measured data, so they are different through the whole year. The peak demands are $42858 \mathrm{~kW}$ and $230693 \mathrm{~kW}$ respectively for electric and thermal power. Fig. 8 shows the heat load duration curve of the district heating network for one year.

The selection and design of the units must be optimized for two different objective functions:

- Test case A: the objective function to be minimized is the total primary energy consumption of the energy systems (cogeneration units and boilers) connected to the network. This objective function is equivalent to minimizing the sum of emissions of $\mathrm{CO}_{2}$ of the units connected to the network since all units use natural gas of fixed composition. The electricity purchased/sold from the electric grid is accounted for in the objective function by computing the associated avoided/caused primary energy consumption of the fossil-fired power plants providing electricity the grid. For the sake of this calculation, we assumed that the average net electric efficiency of the fossil-fired power plants is $44 \%$.

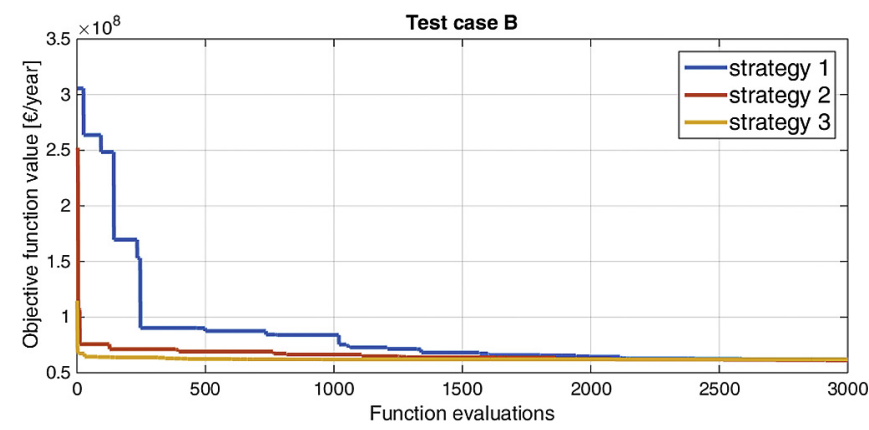

Fig. 12. Convergence plot (objective function value of the best found solution as a function of the number of function evaluations) of the three solution strategies on test case $\mathrm{B}$. 
Table 3

Results for test case A. Results relative to the best solution are reported in bold.

\begin{tabular}{|c|c|c|c|c|c|}
\hline & \multirow[t]{2}{*}{ Strategy 1} & \multicolumn{2}{|l|}{ Strategy 2} & \multicolumn{2}{|l|}{ Strategy 3} \\
\hline & & Relaxed size variables & $\begin{array}{l}\text { Discrete } \\
\text { size variables }\end{array}$ & $\begin{array}{l}\text { Relaxed } \\
\text { size variables }\end{array}$ & $\begin{array}{l}\text { Discrete size } \\
\text { variables }\end{array}$ \\
\hline Total Annual Energy Cost of best solution, kWh/year & 349442697 & 280971332 & 281957190 & 288912488 & 291168890 \\
\hline Total Annual Energy Cost of worst solution, kWh/year & 740479270 & 280960736 & & 288945601 & \\
\hline Total Annual Energy Cost of average solution, kWh/year & 389369598 & 280930593 & & 288924862 & \\
\hline Total average computational time, $\mathrm{s}$ & 14272 & 41855 & & 31882,5 & \\
\hline Average computational time of upper level alg., s & 181 & 338 & & 255 & \\
\hline \multicolumn{6}{|l|}{ Selected Units of the best solution } \\
\hline \multirow[t]{2}{*}{ Combined cycle back pressure, $\mathrm{kW}_{\mathrm{el}}$} & 68254 & 68254 & 68254 & 83084 & 70516 \\
\hline & 68254 & 113292 & 116692 & 98372 & 116692 \\
\hline Combined cycle extraction steam turbine & - & - & - & - & - \\
\hline \multirow[t]{2}{*}{ Gas turbine simple cycle, $\mathrm{kW}_{\mathrm{el}}$} & 5041 & - & - & - & - \\
\hline & 5041 & - & - & - & - \\
\hline \multirow[t]{3}{*}{ Gas turbine post firing, $\mathrm{kW}_{\mathrm{el}}$} & 16357 & - & - & - & - \\
\hline & 16357 & - & - & - & - \\
\hline & 21071 & - & - & - & - \\
\hline Auxiliary boiler $1, \mathrm{~kW}_{\text {th }}$ & 92235 & 24750 & 24750 & 27030 & 27030 \\
\hline Auxiliary boiler $2, \mathrm{~kW}_{\text {th }}$ & - & 52452 & 50760 & 80440 & 74016 \\
\hline Storage, $\mathrm{kW}_{\mathrm{th}}$ & 79738 & 80000 & 80000 & 79953 & 79953 \\
\hline Total annual costs, $€ /$ year & $9.83 \mathrm{E}+07$ & $7.850 \mathrm{E}+07$ & $7.852 E+07$ & $7.982 \mathrm{E}+07$ & $7.996 \mathrm{E}+07$ \\
\hline Investment costs, $€$ & $2.56 \mathrm{E}+08$ & $2.09 \mathrm{E}+08$ & $2.11 E+08$ & $2.14+08$ & $2.19 \mathrm{E}+08$ \\
\hline Operation costs, €/year & $5.58 \mathrm{E}+07$ & $4.38 \mathrm{E}+07$ & $4.34 \mathrm{E}+07$ & $4.42 \mathrm{E}+07$ & $4.36 \mathrm{E}+07$ \\
\hline Total installed electric capacity, kW & 200375 & 181546 & 184946 & 181456 & 187208 \\
\hline Total installed heat capacity, kW & 346515 & 190311 & 190311 & 220523 & 220523 \\
\hline
\end{tabular}

- Test case $B$ : the objective function to be minimized is the total annual cost TAC (operating costs and investment costs) of the plant. This is equivalent to maximize the net present value. are:

The technologies that can be selected for both test cases A and B

- CHP simple cycle gas turbines with heat recovery (GT-SC);

- CHP simple cycle gas turbines with supplementary firing and heat recovery (GT-PF);

- CHP natural gas combined cycles with a back-pressure steam turbine with heat recovery (CC-BP);

- CHP natural gas combined cycles with an extraction-type steam turbine (CC-BE);

- Auxiliary boilers (AB);

\section{- A thermal energy storage system.}

The set of possible sizes for each technology is reported in Table 1. For the gas turbines and the combined cycles, only a subset of the commercially available gas turbine engines have been considered to derive the available sizes as well as the size effects on performance (i.e., electric efficiency) and investment costs. These data have been taken from the commercial software GT PRO [48]. The same software was used to determine the performance maps of the CHP GTs, NGCCs and boilers. In particular, for each technology (i.e., GT-SC, GT-PF, CC$\mathrm{BP}, \mathrm{CC}-\mathrm{BE}$ ), only the performance map of the unit with reference size was calculated and then its normalized shape was extended to model units with different sizes. This assumption is consistent with the idea of considering gas turbines of the same series (e.g., frame F), design layout (e.g., single-shaft)

Table 4

Results for test case B. Results relative to the best solution are reported in bold

\begin{tabular}{|c|c|c|c|c|c|}
\hline & \multirow[t]{2}{*}{ Strategy 1} & \multicolumn{2}{|l|}{ Strategy 2} & \multicolumn{2}{|l|}{ Strategy 3} \\
\hline & & $\begin{array}{l}\text { Relaxed size } \\
\text { variables }\end{array}$ & $\begin{array}{l}\text { Discrete size } \\
\text { variables }\end{array}$ & $\begin{array}{l}\text { Relaxed size } \\
\text { variables }\end{array}$ & $\begin{array}{l}\text { Discrete size } \\
\text { variables }\end{array}$ \\
\hline Total Annual Cost of best solution, €/year & 61208271 & 60892339 & 61290000 & 62036008 & 62040000 \\
\hline Total Annual Cost of worst solution, $€ /$ year & 63920000 & 63695000 & & 62112017 & \\
\hline Total Annual Cost of average solution, $€ /$ year & 62274704 & 61749956 & & 62058352 & \\
\hline Total average computational time, $\mathrm{s}$ & 41030 & 193216 & & 52643 & \\
\hline Average computational time of upper level alg., s & 433 & 1108 & & 651 & \\
\hline \multicolumn{6}{|l|}{ Selected Units of the best solution } \\
\hline Combined cycle back pressure, $\mathrm{kW}_{\mathrm{el}}$ & 68254 & 83259 & 70516 & 70399 & 70516 \\
\hline Combined cycle extraction steam turbine, $\mathrm{kW}_{\mathrm{el}}$ & - & - & - & - & - \\
\hline Gas turbine simple cycle, $\mathrm{kW}_{\mathrm{el}}$ & - & - & - & - & - \\
\hline Gas turbine post firing, $\mathrm{kW}_{\mathrm{el}}$ & - & - & - & - & - \\
\hline Auxiliary boiler $1, \mathrm{~kW}_{\text {th }}$ & 50973 & 43360 & 51300 & 79287 & 79214 \\
\hline Auxiliary boiler $2, \mathrm{~kW}_{\text {th }}$ & 98288 & 95767 & 95767 & 98999 & 98999 \\
\hline Storage, $\mathrm{kW}_{\text {th }}$ & 80000 & 79625 & 79625 & - & - \\
\hline Total Annual Energy Cost, kWh/year & $7.8564 E+08$ & $6.9568 \mathrm{E}+08$ & $7.7854 \mathrm{E}+08$ & $7.9675 \mathrm{E}+08$ & $7.9625 \mathrm{E}+08$ \\
\hline Investment costs, $€$ & 1.07E +08 & $1.19 \mathrm{E}+08$ & $1.09 \mathrm{E}+08$ & $1.09 \mathrm{E}+08$ & $1.09 \mathrm{E}+08$ \\
\hline Operation costs, €/year & $4.34 \mathrm{E}+07$ & $4.12 \mathrm{E}+07$ & $4.32 \mathrm{E}+07$ & $4.39 \mathrm{E}+07$ & $4.39 \mathrm{E}+07$ \\
\hline Total installed electric capacity, kW & 68254 & 83259 & 70516 & 70399 & 70516 \\
\hline Total installed heat capacity, kW & 191786 & 191001 & 191001 & 222148 & 222148 \\
\hline
\end{tabular}


and control mode (e.g., with VIGV) within each technology.

Two additional constraints are included, in order to obtain economically and technically feasible solutions, the maximum investment cost of the overall system (300 M\$) and maximum electricity export to the electric grid (150 MW). The expected life of the energy system is 20 years.

\section{Results}

\subsection{Typical weeks}

First, we calculated the weights necessary for the calculation of the typical weeks. Weights are evaluated as the mean values for the five possible extreme plant configurations, each one based on a single type of technology (GT-SC, GT-PF, CC-BP, CC-BE, AB) and heat storage. For these extreme configurations, the CHP units and the heat storage have been sized with simple design criteria: first, the size of the CHP unit has been matched to the average heat demand of the coldest week of the year increased by $20 \%$, then the size of the heat storage has been adjusted so as to satisfy the peak heat demand (of the coldest week). The weights of the electricity demand, heat demand and ambient temperature profiles obtained by applying the procedure described in Section 5 for each extreme configuration are reported in Table 2 .

According to the weights shown in Table 2, thermal energy demand has the greatest influence on the performance of the system (i.e., total operating cost of the whole year), surprisingly higher (almost double) than that of the electricity demand. This is related to the fact that the scheduling and operation of the units is mainly affected by the heat load profile, even when a thermal storage system is available. Indeed, while a variation of electric power demand can be compensated by purchasing/selling more/ less electricity from/to the electric grid even without the necessity of changing the scheduling solution of the units (which is essentially equivalent to an electric storage system with infinite capacity), a variation of heat demand may require the variation of the scheduling of the units because the thermal storage system has a limited capacity. It is also interesting to note that the weights obtained for the five extreme cases are similar and, when used in the $\mathrm{k}$-means clustering algorithm described in Section 5, all lead to the same typical weeks clustering shown in Fig. 9. Fig. 10 shows the profiles of the thermal and electric power demands of the typical weeks.

In order to calculate the approximation error due to the use of a reduced set of representative weeks, we compared the annual TOC of the 52 original weeks (by optimizing the scheduling of each of them) and the annual TOC estimated considering the 3 typical weeks. When using the different weights for the electric power, thermal power and ambient temperature, the approximation is limited to 3\% (in terms of total operating costs of the whole operating year), while without weights (i.e., using the plain k-means method) the approximation error reaches about $10 \%$.

\subsection{Computational results}

We used the typical weeks obtained with the weighted k-means algorithm for the solution of the design and synthesis optimization problem with the three solution strategies described in Section 4. For all three strategies the maximum number of function evaluations of the black-box function is set to 5100. Each optimization run has been repeated 10 times so as to account for the stochastic nature of the algorithms (eSS and PGS-COM are evolutionary algorithms with random components in the selection of the starting points and search steps). The convergence curves of the best runs (i.e., run which find the best solution) for test case A and B are shown in Figs. 11 and 12 respectively.

The results of the computational tests are shown in Tables 3 and 4. In both test cases, the solutions found by the three strategies are quite different in terms of selected units. Only the size of the heat storage system set at the upper bound is a common feature indicating a clear economic and thermodynamic advantage in installing a large thermal storage system.

For what concerns the energy test case (test case A), strategy 2 finds the best solution, which is composed by two combined cycle with back-pressure steam turbine units, two auxiliary boilers and the heat storage system. The computational time for the solution is 41855s. It should be noticed that the total computational time essentially depends on the calculation time required by the MILP scheduling sub-problem, while the computational time used by the upper level algorithms (eSS and PGS-COM) is practically negligible.

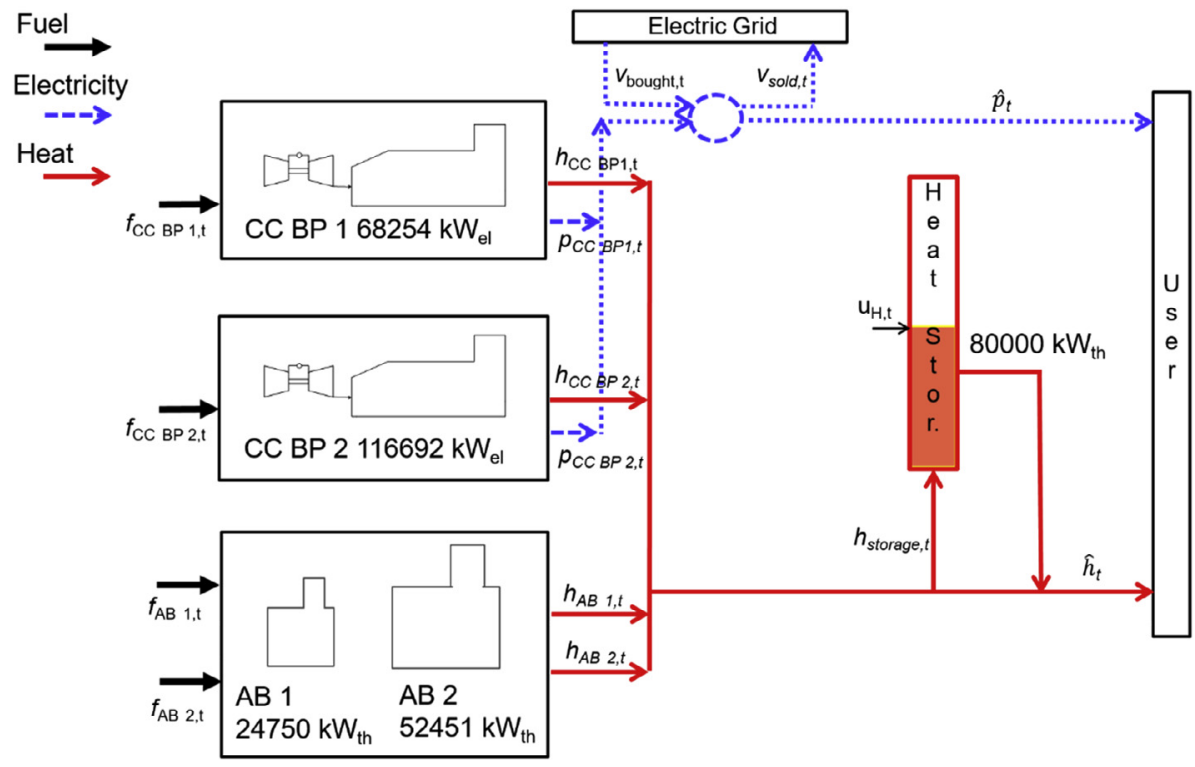

Fig. 13. Conceptual layout and energy flows of the CHP system obtained as best solution for test case A. 


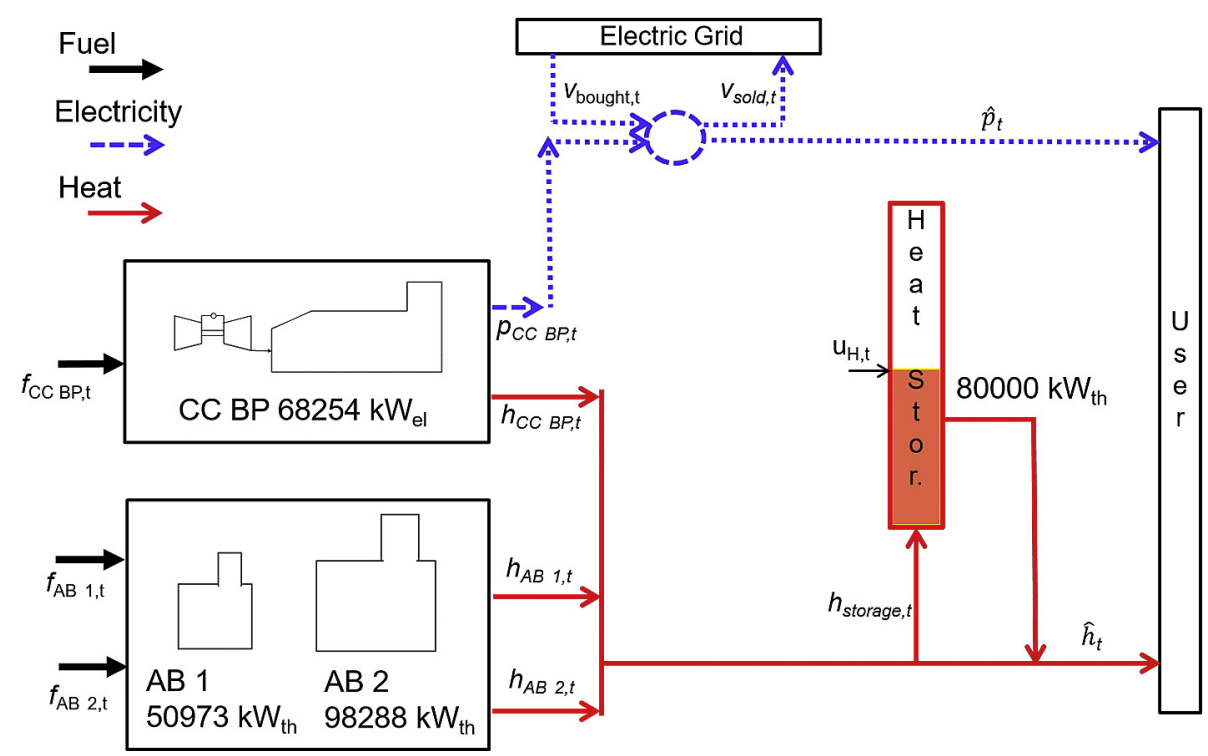

Fig. 14. Conceptual layout and energy flows of the CHP system obtained as best solution for test case B.

Table 5

Comparison of results of best solutions for test case A (best energy cost) and test case B (best economic cost).

\begin{tabular}{|c|c|c|}
\hline & Minimum primary energy consumption & Minimum total annual cost \\
\hline Total installed electric capacity, kW & 184946 & 68254 \\
\hline Total installed heat capacity, kW & 190311 & 191786 \\
\hline Total primary energy consumption (natural gas), GJ/year & 1015056 & 2828304 \\
\hline Investment costs, $€$ & $2.11 \mathrm{E}+08$ & $1.07 \mathrm{E}+08$ \\
\hline Operation costs, $€ /$ year & $4.34 \mathrm{E}+07$ & $4.34 \mathrm{E}+07$ \\
\hline Total annual costs, $€ /$ year & $7.85 \mathrm{E}+07$ & $6.12 \mathrm{E}+07$ \\
\hline
\end{tabular}

Strategy 1 finds a considerably worse solution, due to the limited number of available function evaluations and large number of discrete variables to be optimized. The worst solution obtained with strategy 1 is $110 \%$ suboptimal with respect to the best one of strategy 1 , indicating that many more function evaluations are needed to reach convergence to close-to-optimal solutions. This need is also shown in the convergence plot of Fig. 11 where the eSS algorithm finds an improving solution after about 5050 function evaluations. Strategy 3 very quickly finds a solution which is only $3 \%$ suboptimal with respect to strategy 2 .

As far as the economic test case is concerned (test case B), the three strategies find the same optimal configuration with only differences in sizes of the units. While strategies 1 and 2 find solutions based on a heat storage system with maximum size,

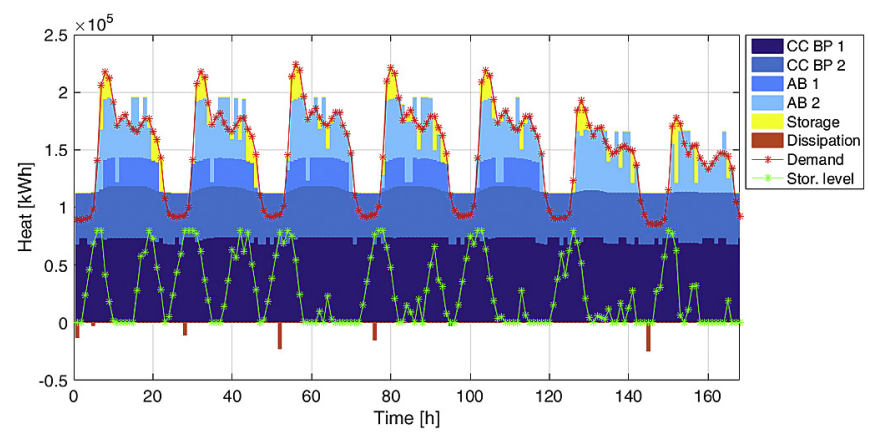

Fig. 15. Plot of the optimal scheduling of the minimum energy consumption design (best solution obtained with strategy 2 for test case A) for typical week 1. strategy 3 finds a suboptimal solution without heat storage and with larger boilers.

Despite the poor performance in the energy case, in the economic optimization strategy 1 finds the best solution. The best solution found by strategy 2 is only slightly $(0.1 \%)$ worse than strategy 1 and it must be noticed that strategy 2 appears to have better reliability and convergence rate (decrease rate of the objective function), as indicated by its average performance and convergence curve in Fig. 12. It is worth noting that the best TAC of the relaxed approach is better than the value found by strategy 1 since the heuristic solution discretization procedure causes a significant increase of TAC. Strategy 3 shows the quickest convergence rate (decrease rate of the objective function shown in Fig. 12) but then gets trapped in a local minima (1.3\% suboptimal in terms of TAC).

The reason why in the economic test case, strategy 1 is particularly effective in obtaining the optimal solution may be the marked superiority of the back-pressure combined cycle unit with respect to the other types of units in terms of capital cost and matching with the electricity and heat demands. This superiority has facilitated the rapid convergence of the MINLP algorithm towards the solution. In absence of such a superior option, the strategy 1 suffers from a low convergence rate due to the number of combinations to be explored, as indicated by the energy test case.

\subsection{Optimal design solutions}

The layouts of the best solutions obtained in the two test cases are represented in Fig. 13 and Fig. 14 respectively while the main features are reported in Table 5. 
(a)

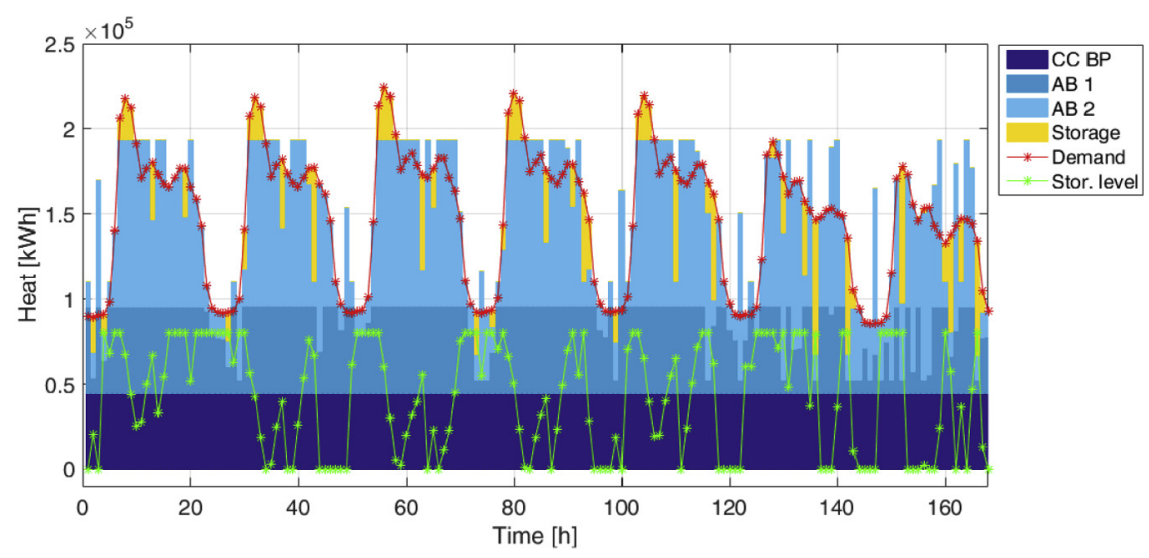

(b)

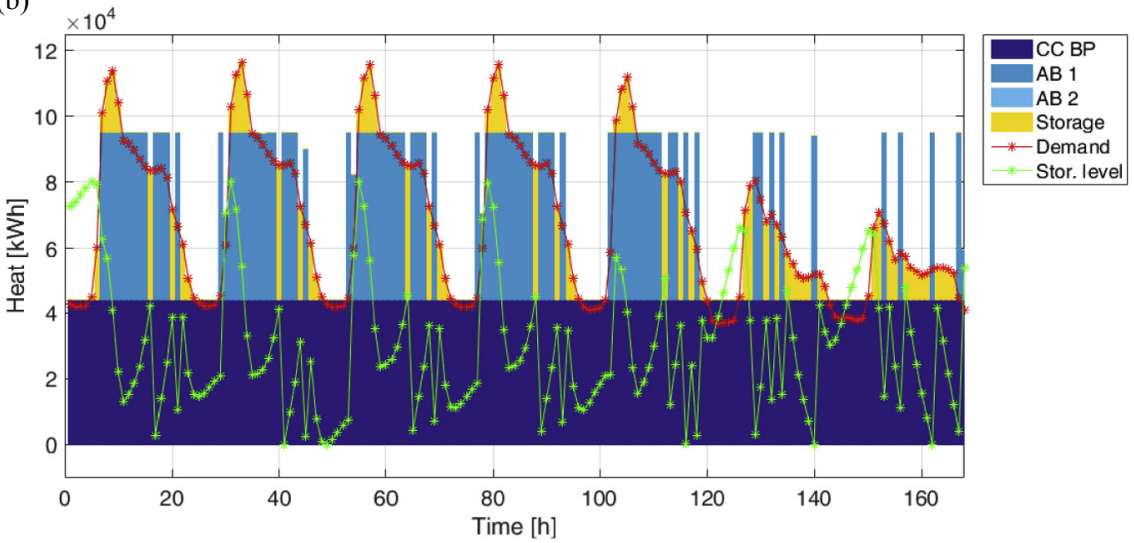

Fig. 16. (a) Plot of the optimal scheduling of the minimum total annual cost design for typical week 1. (b) Plot of the optimal scheduling of the minimum total annual cost design for typical week 2 .

Results indicate that the design optimized for the minimum primary energy consumption allows to save $64 \%$ of primary energy compared to the minimum cost solution, but at the price of a $28 \%$ higher total annual costs. Both solutions feature NGCC with backpressure steam turbine as cogeneration technology which seems to overdo the other technologies, probably for the good compromise between electric efficiency (about $48.7 \%$ at full-load, depending on the size), thermal efficiency (about 32\% at full-load, depending on the size) and costs. Its limits of flexibility in operation (NGCCs with back-pressure steam turbine have only one control variable, the GT load, with the impossibility of independently varying the electric power and the thermal power) are alleviated by the possibility of using the heat storage system. Compared to the minimum cost solution, the minimum primary energy consumption solution adopts a higher installed capacity of $\mathrm{CHP}$ units so as to exploit cogeneration for a larger fraction of the heat demand.

\subsection{Optimal scheduling solutions}

Finally, we present some interesting results of the optimal scheduling for the best design solutions obtained for test case $A$ and B.

\subsubsection{Test case $A$ - optimal scheduling of the best design solution}

Fig. 15 shows the optimal scheduling of the minimum energy consumption design for the typical week with highest heat demand (typical week 1). The maximum thermal power output of the installed CHP units and boilers is not sufficient to meet the peak demand, which is slightly higher. The peak demand is met by exploiting the heat storage system. To this aim, it is important to notice that in the real practice a set of back-up boilers is necessary to meet unexpected peak demands and failures of the installed CHP units. As it can be seen in the plot of the heat balance results for the first typical week (Fig. 15), the two NGCCs essentially run at full load, with minor adjustments aimed at limiting the heat wasted during night (due to the limited size of the heat storage system, a fraction of the cogenerated heat is not stored but rejected to the environment). The fact that a surplus of heat needs to be wasted indicates that a larger storage capacity would further improve the system efficiency, but the size of the installed heat storage system is already at the upper bound specified at the design level. The combined cycles are never shut down because they feature a higher electric efficiency than the average of the electric grid and hence their power export allows to save primary energy. The two boilers are used only during the peak hours of the week days. On Saturdays and Sundays, only the largest one of the two boilers is used. In addition, the heat storage system is always charged during night (off-peak hours) using the NGCCs. However, each day features a different management strategy of the heats storage system. Mondays and Tuesdays have two full charge/ discharge cycles while the other days have a single charge/ discharge cycle (corresponding to the heat demand peak) followed by different management strategies. It would not be 
possible to reproduce this optimal management strategy of the heat storage system if typical days (instead of typical weeks) were considered.

\subsubsection{Test case $B$ - optimal scheduling of the best design solution}

Fig. 16 shows the plot of the optimal scheduling of the minimum total annual cost design for typical weeks 1 and 2 . Similarly to the minimum energy consumption solution, also in this design solution the maximum thermal power output of installed CHP unit and boilers is not sufficient to meet the peak demand, which is slightly higher. The peak demand (occurring in the first typical week) is met by exploiting the heat storage system, as shown in Fig. 16(a). The combined cycle unit always runs at full load. As shown in Fig. 16(a), in the typical week 1 the heat storage system needs to be charged by the boilers when the heat demand is below the installed capacity, not only during day but also during night (the larger boiler is turned on during night to charge the heat storage so as to have it ready for the following morning). In the typical week 2 , that features lower heat demands than typical week 1 , only the smallest of the two installed boilers is turned on and the peak demand is met by exploiting the heat storage system (see Fig. 16(b)). The NGCC runs always at full load. Every day has a different optimal strategy to manage the heat storage system. In particular, during weekdays, the heat storage system is charged during the night by the combined cycle and by turning on the smallest boiler at full load a couple of hours before the morning peak of heat demand. The heat storage is also used to cover the second peak demand of the day, occurring in the evening. In the morning of Friday, the storage is not charged to the full load and, on the other hand, it is not fully consumed (by switching on the boiler also during late evening) so as to preserve its charge level for the weekend. This strategy allows to minimize the use of the boiler during the weekend.

\section{Conclusions}

This paper presents an effective approach for the design and synthesis of Combined Heat and Power systems for district heating networks. The approach allows to optimize the system design (selection and sizing of the units) while considering and optimizing the multi-period operation for an expected set of typical weeks. Operational flexibility of the selected units, the actual nonlinear effects of the unit size on the performance curve and costs are accounted for with detailed models. The computational results obtained for an industrial-scale test problem (a district heating network featuring up to 14 potential generation units) show that:

1. When determining the profiles of the typical weeks with the kmeans algorithm, it is not proper to give the same weight to the approximation errors of the different profiles (heat demand, electric demand and ambient temperature). Surprisingly, the heat demand profile has a larger effect, almost double than the electric demand profile, on the objective function value (total operating cost of the whole year). This is related to the fact that the scheduling and operation of the units is mainly affected by the heat load profile, even when a thermal storage system is available. Thanks to the proper definition of the relative weights of the three profiles, the approximation error associated to the use of the typical weeks compared to the actual yearly profiles can be reduced from 10\% to 3\% (in terms of total operating costs of the whole operating year).

2. As far as the solutions strategy to tackle the MINLP design problem, strategy 2 (using binary variables to select the installed units, a continuous relaxation of the sizes, the eSS algorithm to deal with the design MINLP, and then a heuristic procedure to find the closest available discrete size) achieves the best performance in terms of trade-off between convergence rate (decrease rate of the objective function value), robustness (capability of reaching good solutions at each run) and quality of the solutions. Although the solution quality of the design solutions found the by the upper level algorithm (eSS) cannot be guaranteed as the algorithm is meta-heuristic, the small difference registered between the worst and best run solution (over the 10 runs executed) indicates a good reliability of the proposed method. In general, the strategy with no relaxations of the integer variables suffers from a low convergence rate due to the limited number of available function evaluations and large number of discrete variables to be optimized, as indicated by the energy test case. It is worth noting that the continuous relaxation of the discrete variables would be even more advantageous if more units with discrete sizes (i.e., GTs and NGCCs) were included in the list of possible options.

3. For the presented test cases, the optimization runs (solution of both lower level and upper level problems) adopting the bounding procedure devised in Section 4.3 allows to save about $30 \%$ of the computational time with respect to runs in which the procedure is not used. Moreover, the design solutions sampled by the upper-level evolutionary algorithm (eSS and PGS-COM) could be evaluated in parallel on the available processors of the workstation, with an almost proportional decrease of computational time.

4. For the test case considered in this study, results indicate that NGCCs with back-pressure steam turbine is the best cogeneration technology in terms of both primary energy saving and economic profitability. They seem to overdo the other technologies, probably for the good compromise between electric efficiency (about $48.7 \%$ at full-load, slightly increasing with the size), thermal efficiency (about $32 \%$ at full-load) and costs. Its limits of flexibility in operation are alleviated by the possibility of using the heat storage system. Compared to the minimum cost solution, the minimum primary energy consumption solution adopts a higher installed capacity of CHP units so as to exploit cogeneration for a larger fraction of the heat demand. In both cases, the optimal design solution has an installed thermal capacity lower than the peak heat demand, and manages the heat storage system throughout the whole week so as to meet the peak heat demand. Interestingly, the optimal management strategy of the heat storage system changes day by day within the week as a result of the different heat demand profiles of the weekdays and weekend. This could not have been modelled if typical days were considered instead of typical weeks. The minimum primary energy consumption design allows to save $64 \%$ of primary energy compared to the minimum cost solution, but with a $28 \%$ increase of total annual cost.

In conclusion, the proposed algorithm is an effective design tool that can be used for the design of efficient systems of CHP units.

\section{Acknowledgments}

The authors acknowledge LEAP (Laboratorio Energia Ambiente Piacenza) and the "Efficity" project (grant number CUP E38I16000130007), co-funded by Regione Emilia Romagna in the framework of the Actions 1.2 and 1.3 of 2011-2013 Regional Energy Plan and in coherence with the Action 1.2.2 of 2014-2020 ERDF ROP, for providing financial support. 


\section{Nomenclature}

Sets and parameters

$T=\left\{1,2, \ldots, T_{\max }\right\}$ set of hours of the time period (day, week or year)

All set of all units

$C A$ set of units which are available from a catalog with discrete sizes

$\mathrm{CU}$ set of units which are available in customized sizes

$\widehat{T}_{0, \mathrm{t}}, t \in T$ ambient temperature at time $t$

$\widehat{p}_{\mathrm{t}}, t \in T$ required electric energy at time $t$

$\widehat{h}_{\mathrm{t}}, t \in T$ required heat at time $t$

$u_{\mathrm{h}, \text { losses }}$ heat storage percentage losses

CC cost of carry or capital carrying charge rate

$X_{\mathrm{D} \text { min }, i}, i \in C U$ lower bound for the value of the size of customizable unit $i$

$X_{\mathrm{D} \text { max }, i}, i \in C U$ upper bound for the value of the size of customizable unit $i$

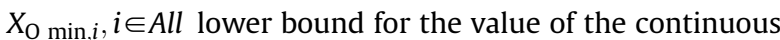
independent operative variables of unit $i$

$X_{\mathrm{O} \text { max }, i}, i \in$ All upper bound for the value of the continuous independent operative variables of unit $i$

$\mathrm{Y}_{i, 1}, Y_{i, 2}, Y_{i, 3}, \ldots, i \in C A$ available discrete values of sizes for catalog unit $i$

$l_{\mathrm{H}, \text { storage,min }}$ lower bound for the value of the maximum capacity of the heat storage system

$l_{\mathrm{H} \text {,storage,max }}$ upper bound for the value of the maximum capacity of the heat storage system

Decision variables

$f_{\mathrm{i}, \mathrm{t}}, i \in$ All and $t \in T$ fuel mass flow rate to unit $i$ at time $t$

$p_{\mathrm{i}, \mathrm{t}}, i \in$ All and $t \in T$ electric power generated by unit $i$ at time $t$

$h_{\mathrm{i}, \mathrm{t}}, i \in$ All and $t \in T$ thermal power generated by unit $i$ at time $t$

$u_{H, \mathrm{t}}, t \in T$ level of heat accumulated in the storage system at time $t$

$v_{\text {sold }, \mathrm{t}}, t \in T$ electric power sold to the grid at time $t$

$v_{\text {bought }, \mathrm{t}}, t \in T$ electric power bought from the grid at time $t$

$z_{\mathrm{O}, \mathrm{i}, \mathrm{t}}, i \in$ All and $t \in T$ binary variable used to denote the on/off

status of unit $i$ at time $t$

$x_{0, i, t}, i \in A l l$ and $t \in T$ continuous independent operative variables (e.g., load) of unit $i$ at time $t$

$z_{\mathrm{D}, \mathrm{i}}, i \in$ Allbinary variable used to $\operatorname{select}\left(z_{D, i}=1\right)$ or not $\left(z_{D, i}=0\right)$ unit $i$

$y_{\mathrm{D}, i}, i \in C A$ integer variable used to select the discrete size of unit $i$

$x_{\mathrm{D}, i}, i \in C U$ continuous variable used to select the customized size of unit $i$

$l_{H, \text { storage }}$ continuous variable used to select the maximum capacity of the heat storage system

\section{Acronyms}

$\mathrm{AB} \quad$ Auxiliary boilers

CC-BE Natural gas combined cycles with a bottoming extractiontype steam turbine with heat recovery

CC-BP Natural gas combined cycles with a bottoming backpressure steam turbine with heat recover

GT-PF Simple cycle gas turbines with post firing and heat recovery

GT-SC Simple cycle gas turbines with heat recovery

LB Lower Bound

MILP Mixed Integer Linear Problem

MINLP Mixed Integer Non-Linear Problem

TAC Total Annual Cost

TOC Total Operating Cost

\section{References}

[1] Kerr T. Cogeneration and district heating. IEA; 2009. www.iea.org.

[2] Pohl E, Diarra D. Assessment of primary energy savings by means of CHP systems in domestic energy supply. Appl Therm Eng 2014;71(2):830-7.

[3] Ommen T, Markussen WB, Elmegaard B. Lowering district heating temperatures - impact to system performance in current and future Danish energy scenarios. Energy 2016;94:273-91.

[4] Christidis A, Koch C, Pottel L, Tsatsaronis G. The contribution of heat storage to the profitable operation of combined heat and power plants in liberalized electricity markets. Energy 2012;41(1):75-82.

[5] Bischi A, Taccari L, Martelli E, Amaldi E, Manzolini G, Silva P, et al. A rollinghorizon MILP optimization method for the operational scheduling of trigeneration systems with incentives. In: Proceedings of ECOS international conference; 2015. ISBN 978-2-9555539-0-9, Pau, France, July 2015.

[6] Raine R, Sjarifi VN, Swithenbank J. Optimisation of combined heat and power production for buildings using heat storage. Energy Convers Manag 2014;87: 164-74.

[7] Verda V, Colella F. Primary energy savings through thermal storage in district heating networks. Energy 2011;36(7):4278-86.

[8] Lund H, Werner S, Wiltshire R, Svendsen S, Thorsen JE, Hvelplund F, et al. 4th generation district heating (4GDH) integrating smart thermal grids into future sustainable energy systems. Energy 2014;68:1-11.

[9] Frangopoulos CA, von Spakovsky MR, Sciubba E. Brief review of methods for the design and synthesis optimization of energy systems. Int J Appl Thermodyn 2002;5(4):151-60.

[10] Bischi A, Taccari L, Martelli E, Amaldi E, Manzolini G, Silva P. A detailed MILP optimization model for combined cooling, heat and power system operation planning. Energy 2014;74:12-26.

[11] Fazlollahi S, Bungener SL, Mandel P, Becker G, Maréchal F. Multi-objectives, multi-period optimization of district energy systems: I. Selection of typical operating periods. Comput Chem Eng 2014;65:54-66.

[12] Taccari L, Amaldi E, Martelli E, Bischi A. Short-term planning of cogeneration power plants: a comparison between MINLP and piecewise-linear MILP formulations. Comput Aided Chem Eng 2015;37:2429-34.

[13] Cho W, Lee K-S. A simple sizing method for combined heat and power units. Energy 2014;65:123-33.

[14] EPRI (Electric Power Research Institute). EPRI report TR-102275. TAG e technical assessment guide (electric supply), vol. 1; 1993. Rev. 7.

[15] Phung DL. Theory and evidence for using the economy-of-scale law in power plant economics, report ORNL/TM-10195. 1987. web.ornl.gov/info/reports/ 1987/3445602624186.pdf (accessed 24 July 2016).

[16] Gas Turbine World. Gas turbine world handbook 2014-2015, vol. 31; 2015. http://www.gasturbineworld.com/order-now.html (accessed 27 July 2016).

[17] Bejan A, Lorent S, Yilba BS, Sahin AZ. The effect of size on efficiency: power plants and vascular designs. Int J Heat Mass Transf 2011;54:1475-81.

[18] Lozza G. Bottoming steam cycles for combined steam gas power plants: a theoretical estimation of steam turbines performances and cycle analysis. In: Proceedings of the 1990 ASME cogen-turbo symposium New Orleans, USA; 1990. p. 83-92.

[19] Hoval. Industrial Boilers: complete system solutions for steam and hot water applications. 2016. http://www.hovalpartners.com/products-solutions/ solutions/industrial-boiler-solutions (accessed 26 July 2016).

[20] Mitra S, Sun L, Grossmann IE. Optimal scheduling of industrial combined heat and power plants under time-sensitive electricity prices. Energy 2013;54: 194-211.

[21] Wolsey LA. Integer programming. Wiley; 1998. ISBN: 978-0-471-28366-9.

[22] IBM ILOG CPLEX optimizer. http://www-01.ibm.com/software/commerce/ optimization/cplex-optimizer/. (accessed 26 July 2016)

[23] Gurobi optimizer, version 6.0. Available online: http://www.gurobi.com. (accessed 26 July 2016).

[24] FICO XPRESS optimizer, version 7.8. http://www.fico.com/en/products/ficoxpress-optimization-suite. (accessed 26 July 2016).

[25] Iyer R, Grossmann I. Synthesis and operational planning of utility systems for multiperiod operation. Comput Chem Eng 1998;22(7-8):979-93.

[26] Maréchal F, Kalitventzeff B. Targeting the integration of multi-period utility systems for site scale process integration. Appl Therm Eng 2003;23(14): 1763-84.

[27] Maréchal F, Kalitventzeff B. Process integration: selection of the optimal utility system. Comput Chem Eng 1998;22(Supplement 1):S149-56. 0.

[28] Arcuri P, Florio G, Fragiacomo P. A mixed integer programming model for optimal design of trigeneration in a hospital complex. Energy 2007;32(8): 1430-47.

[29] Yokoyama R, Hasegawa Y, Ito K. A MILP decomposition approach to large scale optimization in structural design of energy supply systems. Energy Convers Manag 2002;43(6):771-90.

[30] Yokoyama R, Ito K. Optimal design of gas turbine cogeneration plants in consideration of discreteness of equipment capacities. J Eng Gas Turbines Power 2006;128(2):336-43.

[31] Yokoyama R, Shinano Y, Taniguchi S, Ohkura M, Wakui T. Optimization of energy supply systems by MILP branch and bound method in consideration of hierarchical relationship between design and operation. Energy Convers 
Manag 2015;92:92-104.

[32] Aguilar O, Perry SJ, Kim J-K, Smith R. Design and optimization of flexible utility systems subject to variable conditions: part 2: methodology and applications. Chem Eng Res Des 2007;85(8):1149-68.

[33] Buoro D, Casisi M, De Nardi A, Pinamonti P, Reini M. Multicriteria optimization of a distributed energy supply system for an industrial area. Energy 2013;58: $128-37$.

[34] Omu A, Choudhary R, Boies A. Distributed energy resource system optimisation using mixed integer linear programming. Energy Policy 2013;61:249-66.

[35] Bracco S, Dentici G, Siri S. Economic and environmental optimization model the the design and the operation of a combined heat and power distributed generation system in an urban area. Energy 2013;55:1014-24.

[36] Bracco S, Dentici G Siri S. DESOD: a mathematical programming tool to optimally design a distributed energy system. Energy 2016;100:298-309.

[37] Yang Y, Zhang S, Xiao Y. Optimal design of distributed energy resource systems coupled with energy distribution networks. Energy 2015;85:433-48.

[38] Rubio-Maya C, Javier Uche J, Martínez A. Sequential optimization of a polygeneration plant. Energy Convers Manag 2011;52(8-9):2861-9.

[39] Grossmann IE, Viswanathan J, Vecchieti A. DICOPT solver. Washington, DC: GAMS Development Corporation; 2009.

[40] Rosenthal RE. GAMS - a user's guide. Washington, DC, USA: GAMS Development Corporation; 2008.

[41] Fazlollahi S, Becker G, Maréchal F. Multi-objectives, multi-period optimization of district energy systems: II. Daily thermal storage. Comput Chem Eng 2014;71:648-62.

[42] Fazlollahi S, Becker G, Maréchal F. Multi-objectives, multi-period optimization of district energy systems: III. Distribution networks. Comput Chem Eng 2014;66:82-97.

[43] Stojiljković MM, Stojiljković MM, Blagojević BD. Multi-objective combinatorial optimization of trigeneration plants based on metaheuristics. Energies 2014;7:8554-81.

[44] Zidan A, Gabbar HA, Eldessouky A. Optimal planning of combined heat and power systems within microgrids. Energy 2015;93:235-44.

[45] Martelli E. Numerical optimization of heat recovery steam cycles for highly integrated energy systems. Ph.D. Thesis. Italy: Department of Energy, Politecnico di Milano; 2010

[46] Dvořák M, Havel P. Combined heat and power production planning under liberalized market conditions. Appl Therm Energy 2012;43:163-73.
[47] D'Ambrosio C, Lodi A, Martello S. Piecewise linear approximation of functions of two variables in MILP models. Operations Res Lett 2010;38(1):39-46.

[48] Thermoflow. GT PRO Version 25, software for the performance and cost assessment of gas turbines and combined cycles. Thermoflow Inc; 2016.

[49] Sahinidis NV. BARON 14.4.0: global optimization of mixed-integer nonlinear programs, user's manual. 2014.

[50] Egea JA, Marti R, Banga JR. An evolutionary method for complex-process optimization. Comput Operations Res 2010;37(2):315-24

[51] Martelli E, Amaldi E. PGS-COM: a hybrid method for constrained non-smooth black-box optimization problems: brief review, novel algorithm and comparative evaluation. Comput Chem Eng 2014;63:108-39.

[52] Laguna M, Martí R. Scatter search: methodology and implementations in C. Boston: Kluwer Academic Publishers; 2003.

[53] Glover F, Laguna M, Martí R. Scatter search and path relinking: foundations and advanced designs. In: Onwubolu GC, Babu BV, editors. New optimization techniques in engineering. Springer-Verlag; 2004. pp.87-100.

[54] $\mathrm{Hu}$ X, Eberhart R. Solving constrained nonlinear optimization problems with particle swarm optimization. In: Proceedings of the 6th world multiconference on systematics, cybernetics and informatics, Orlando, USA; 2002.

[55] Lewis RM, Shepherd A, Torczon V. Implementing generating set search methods for linearly constrained minimization. SIAM J Sci Comput 2007;29(6):2507-30.

[56] Andersson J. Multiobjective optimization in engineering design: application to fluid power systems. Ph.D. thesis. Sweden: Department of Mechanical Engineering, Linköpings universitet; 2001.

[57] Nord LO, Martelli E, Bolland O. Weight and power optimization of steam bottoming cycle for offshore oil and gas installations. Energy 2014;76:891-8.

[58] Gatti M, Martelli E, Maréchal F, Consonni S. Multi-objective optimization of a

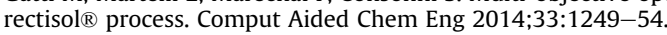

[59] Capra F, Martelli E. Numerical optimization of CHP organic rankine cycles Part B: simultaneous design \& part-load optimization. Energy 2015;90: 329-43.

[60] Hastie T, Tibshirani R, Friedman J. The elements of statistical learning: data mining, inference and prediction. 2 ed. Springer; 2008.

[61] Dominguez-Munoz F, Cejudo-Lopez JM, Carrillo-Andres A, Gallardo-Salazar M. Selection of typical demand days for CHP optimization. Energy Build 2011;43(11):3036-43. 